\title{
La variété des équations surstables
}

Bulletin de la S. M. F., tome 128, no 4 (2000), p. 497-528

<http://www.numdam.org/item?id=BSMF_2000_128_4_497_0>

(c) Bulletin de la S. M. F., 2000, tous droits réservés.

L'accès aux archives de la revue «Bulletin de la S. M. F. » (http: //smf.emath.fr/Publications/Bulletin/Presentation.html) implique l'accord avec les conditions générales d'utilisation (http://www.numdam.org/ conditions). Toute utilisation commerciale ou impression systématique est constitutive d'une infraction pénale. Toute copie ou impression de ce fichier doit contenir la présente mention de copyright.

\section{Numdam}


Bull. Soc. math. France,

128,2000 , p. $497-528$.

\title{
LA VARIÉTÉ DES ÉQUATIONS SURSTABLES
}

\author{
PAR GuY WALLET $(*)$
}

\begin{abstract}
RÉSUMÉ. - On se propose de donner une description géométrique de l'ensemble des équations différentielles d'ordre 1 singulièrement perturbées dans le champ complexe qui admettent des solutions surstables, c'est-à-dire des solutions possédant un développement asymptotique en puissance du petit paramètre $\varepsilon$ dont les coefficients sont des fonctions analytiques sur un même ouvert de $\mathbb{C}$ indépendant de $\varepsilon$. Cette description met en évidence une sorte de structure de variété dans une limite inductive d'espaces de Banach dont les éléments sont des séries formelles à coefficients holomorphes.
\end{abstract}

Abstract. - The MANifold of OVERStable EQUATIOns. - Our purpose is to give a geometric description of the set of singularly perturbed differential equations of order 1 in the complex domain which have overstable solutions, that is to say solutions with an asymptotic expansion in power of the small parameter $\varepsilon$ whose coefficients are analytic functions all defined on the same open set of $\mathbb{C}$. This description shows a kind of manifold structure in some inductive limite of Banach spaces whose elements are formal power series with holomorphic coefficients.

\section{Introduction}

Le cadre le plus simple dans lequel on peut définir la notion de solution surstable [4], [6], [16] est celui d'une équation différentielle singulièrement perturbée $\left(\mathcal{E}_{F}\right)$ dans le champ complexe de la forme

$$
\varepsilon \frac{\partial u}{\partial \xi}=F(\xi, u, \varepsilon)
$$

où dans un premier temps, $F$ désigne une fonction analytique à valeurs dans $\mathbb{C}$ et définie sur un voisinage d'un point $\left(\xi_{0}, u_{0}, 0\right)$ dans $\mathbb{C}^{3}$, de sorte que $F\left(\xi_{0}, u_{0}, 0\right)=0$.

(*) Texte reçu le 26 juillet 1999, accepté le 16 novembre 1999.

G. WAllet, Université de La Rochelle, Pôle Sciences et Technologies, Laboratoire de Mathématiques, avenue M. Crépeau, 17042 La Rochelle CEDEx (France).

Email : gwallet@univ-lr.fr.

Mots clés : perturbation singulière, point tournant, développement asymptotique, Gevrey, solution surstable.

Classification mathématique par matières : $34 \mathrm{C}, 34 \mathrm{D}, 34 \mathrm{E}, 40$.

BULLETIN DE LA SOCIÉTÉ MATHÉMATIQUE DE FRANCE

(C) Société mathématique de France 
On suppose que l'ensemble analytique

$$
\mathcal{L}=\left\{(\xi, u) \in \mathbb{C}^{2} ; F(\xi, u, 0)=0\right\}
$$

(appelé l'ensemble lent de $\left(\mathcal{E}_{F}\right)$ ) contient une courbe lisse $\mathcal{C}$ (appelée une courbe lente de $\left(\mathcal{E}_{F}\right)$ ) qui est le graphe d'une fonction analytique $\varphi_{0}$ définie au voisinage de $\xi_{0}$ telle que $\varphi_{0}\left(\xi_{0}\right)=u_{0}$. La fonction $\varphi_{0}$ est donc une solution de l'équation réduite $\left(\mathcal{E}_{F}^{0}\right)$

$$
0=F(\xi, u, 0)
$$

obtenue à partir de $\left(\mathcal{E}_{F}\right)$ en faisant $\varepsilon=0$. Cette dernière équation a changé de nature : ce n'est plus une équation différentielle mais une équation implicite. C'est pour cela que l'on parle de perturbation singulière.

De manière informelle, une solution surstable de $\left(\mathcal{E}_{F}\right)$ est une solution $\varphi$ de $\left(\mathcal{E}_{F}\right)$ qui approxime la solution $\varphi_{0}$ de l'équation réduite $\left(\mathcal{E}_{F}^{0}\right)$ sur un voisinage de $\xi_{0}$ dans $\mathbb{C}$ indépendant de $\varepsilon$.

Plus précisément, une solution surstable de l'équation $\left(\mathcal{E}_{F}\right)$ subordonnée à la courbe lente $\mathcal{C}$ est une famille analytique $\left(\varphi_{\varepsilon}\right)$ de fonctions telle que :

- il existe un voisinage $V$ de $\xi_{0}$ dans $\mathbb{C}$ indépendant de $\varepsilon$ sur lequel chaque $\varphi_{\varepsilon}$ est définie et analytique;

- pour tout $\varepsilon$ et pour tout $\xi \in V$, on a $\varepsilon \mathrm{d} \varphi_{\varepsilon} / \mathrm{d} \xi(\xi)=F\left(\xi, \varphi_{\varepsilon}(\xi), \varepsilon\right)$;

- lorsque $\varepsilon$ tend vers 0 , la famille de fonctions $\left(\varphi_{\varepsilon}\right)$ converge vers $\varphi_{0}$ uniformément sur les compacts de $V$.

Pour l'instant, rien n'est dit sur la manière dont $\varepsilon$ tend vers 0 .

Pour mener à bien l'étude, il est nécessaire de faire une hypothèse générique sur la nature de la singularité $\left(\xi_{0}, u_{0}\right)$ de l'ensemble $\mathcal{L}$. On suppose que le germe en $\xi_{0}$ de la fonction holomorphe $\xi \mapsto \partial / \partial u F\left(\xi, \varphi_{0}(\xi), 0\right)$ est non nul. Quitte à restreindre le voisinage $V$ de $\xi_{0}$, on peut supposer que cette fonction est de la forme $\xi \mapsto\left(\xi-\xi_{0}\right)^{p} \chi(\xi)$ avec $p \in \mathbb{N}$ et $\chi$ une fonction holomorphe sans zéros dans $V$. On dit que $p$ est l'indice de fugacité [16] des solutions surstables de $\left(\mathcal{E}_{F}\right)$ subordonnées à $\mathcal{C}$.

Lorsque l'indice de fugacité $p$ est non nul, le point $\xi_{0}$ est ce que l'on appelle un point tournant et il n'existe pas en général de solutions surstables subordonnées à $\mathcal{C}$. Dans ces conditions, il faut «rajouter des paramètres» à la fonction $F$ afin de les faire apparaître. De manière plus précise, on remplace $F(\xi, u, \varepsilon)$ par $F(\xi, u, a, \varepsilon)$ où $a$ est un paramètre multidimensionnel appartenant à $\mathbb{C}^{p}$. Sous une hypothèse supplémentaire de transversalité $a d$ hoc, on peut prouver [3], [5], [15] l'existence de solutions surstables pour l'équation différentielle plus générale

$$
\varepsilon \frac{\partial u}{\partial \xi}=F(\xi, u, a(\varepsilon), \varepsilon)
$$

TOME $128-2000-\mathrm{N}^{\circ} 4$ 
dans laquelle $a(\varepsilon)$ désigne une fonction analytique convenable définie sur un secteur ouvert de $\mathbb{C}$ de sommet 0 , à valeurs dans $\mathbb{C}^{p}$ et admettant un développement asymptotique Gevrey d'ordre 1 lorsque $\varepsilon$ tend vers 0 . De plus, on montre que les solutions surstables que l'on obtient dans ce cas admettent elles-même un développement asymptotique Gevrey d'ordre 1.

Ces résultats montrent que le problème initial était mal posé. En effet, pour que le phénomène souhaité se présente, d'une part il faut supposer que la fonction $F$ appartient à un espace de fonctions Gevrey, d'autre part, il faut pouvoir se déplacer dans cet espace pour choisir $F$ convenablement.

Le but du présent travail est de changer de point de vue par rapport à l'approche «à paramètres », en donnant, dans des espaces fonctionnels adaptés, une description de l'ensemble des fonctions $F$ pour lesquelles le phénomène de surstabilité se présente. Autrement dit, on se propose de remplacer une famille de fonctions à $p$ paramètres par l'espace de «toutes》 les fonctions. Ainsi, on espère donner une explication géométrique globale à l'hypothèse de transversalité et au nombre $p$ des paramètres invoquées ci-dessus en mettant en évidence une structure de sous-variété différentiable de classe $C^{1}$ transverse à un sous-espace de dimension $p$ sur une algèbre de séries formelles Gevrey.

Les méthodes utilisées sont celles de l'asymptotique exacte [1], [8], [9], [11], [14], l'attention principale étant portée aux séries formelles Gevrey naturellement associées au problème. Plus précisément, les «bons espaces》 s'avèrent être des espaces de séries formelles à coefficients holomorphes à croissance Gevrey, véritables intermédiaires entre les espaces de séries formelles «pures» et les espaces de fonctions analytiques. Cette étude doit beaucoup aux travaux précédents [6], [12], [13] avec la systématisation qui y est faite de l'outil des normes de Nagumo pour un contrôle Gevrey adapté aux problèmes de perturbation singulière.

Afin de bien dégager les idées générales des développements techniques, on a choisi de rester dans le cadre simple d'une équation différentielle scalaire et de l'ordre Gevrey 1. En suivant le point de vue développé dans [6], il est clair qu'une étude analogue pourrait être menée dans le cas plus général d'un système différentiel avec un ordre Gevrey arbitraire.

Dans le paragraphe suivant, on donne une définition précise de diverses propriétés dites de surstabilité. Malheureusement, il n'est pas possible d'énoncer rapidement les résultats principaux relatifs à l'ensemble des équations surstables; ce sont les théorèmes 2 et 3 du sous-paragraphe 7.1. En effet, la formulation de ces derniers nécessite d'utiliser plusieurs familles d'espaces de Banach dont l'introduction et l'étude est menée sur plusieurs paragraphes. D'ailleurs, ce travail peut aussi être conçu comme une investigation des propriétés, dans ces espaces, de certains opérateurs naturellement associés à une équation différentielle singulièrement perturbées. 


\section{Solutions et équations surstables}

Étant donné un ouvert $U$ de $\mathbb{C}$, on note $\mathcal{O}(U)$ l'ensemble des fonctions analytiques de $U$ dans $\mathbb{C}$ et, pour tout $m \in \mathbb{N}, \mathbb{C}[x]_{m}$ désigne l'ensemble des polynômes à coefficients dans $\mathbb{C}$ de degré strictement inférieur à $m$. Tous les secteurs considérés dans cet article sont ouverts, ont pour sommet 0 et sont des sous-ensemble de $\mathbb{C}$. On se donne :

- un voisinage ouvert $U_{0}$ d'un point $\left(\xi_{0}, u_{0}\right)$ dans $\mathbb{C}^{2}$, un secteur $S_{0}$ et l'ouvert $W_{0}=U_{0} \times S_{0}$ de $\mathbb{C}^{3}$

- un voisinage ouvert $V_{0}$ de $\xi_{0}$ dans $\mathbb{C}$ et une fonction $\varphi_{0} \in \mathcal{O}\left(V_{0}\right)$ telle que $\varphi_{0}\left(\xi_{0}\right)=u_{0}$ et que $\left(\xi, \varphi_{0}(\xi)\right) \in U_{0}$ pour tout $\xi \in V_{0}$;

- une fonction $\chi$ définie, holomorphe et sans zéro sur un voisinage de 0 dans $\mathbb{C}$;

- un nombre $p \in \mathbb{N}$.

On désigne par courbe lente le graphe $\mathcal{C}$ de la fonction $\varphi_{0}$.

On considère l'ensemble $\mathcal{A}\left(W_{0}, \varphi_{0}, \chi, p\right)$ des fonctions analytiques $F: W_{0} \rightarrow \mathbb{C}$ telles que :

- $F$ admet un développement asymptotique $\sum_{n \geq 0} F_{n} \varepsilon^{n} \in \mathcal{O}\left(W_{0}\right)[[\varepsilon]]$ qui est Gevrey d'ordre 1 lorsque $\varepsilon$ tend vers 0 ; cela signifie que pour tout secteur $S$ tel que $^{(1)} S \Subset S_{0}$, il existe des constantes réelles positives $A$ et $B$ de sorte que, pour tout $N \in \mathbb{N}^{*}$ et pour tout $(\xi, u, \varepsilon) \in U_{0} \times S$

$$
\left|F(\xi, u, \varepsilon)-\sum_{n=0}^{N-1} F_{n}(\xi, u) \varepsilon^{n}\right| \leq A B^{N} N !|\varepsilon|^{N}
$$

- pour tout $\xi \in V_{0}$, on a $F_{0}\left(\xi, \varphi_{0}(\xi)\right)=0$;

- pour tout $\xi \in V_{0}$, on a $\partial / \partial u F_{0}\left(\xi, \varphi_{0}(\xi)\right)=\left(\xi-\xi_{0}\right)^{p} \chi\left(\xi-\xi_{0}\right)$.

$\grave{A}$ tout $F \in \mathcal{A}\left(W_{0}, \varphi_{0}, \chi, p\right)$, on associe l'équation différentielle $\left(\mathcal{E}_{F}\right)$ de la forme (1).

DÉfinition 1. - Soit $F \in \mathcal{A}\left(W_{0}, \varphi_{0}, \chi, p\right)$. Étant donnée une direction $\theta d u$ secteur $S_{0}$, une solution $\mathcal{C}$-surstable dans la direction $\theta$ de l'équation $\left(\mathcal{E}_{F}\right)$ est une fonction analytique $\varphi: V \times S_{\theta}^{\prime} \rightarrow \mathbb{C}$ où $V$ est un voisinage ouvert de $\xi_{0}$ dans $V_{0}, S_{\theta}^{\prime}$ est un sous-secteur de $S_{0}$ contenant la direction $\theta$ de sorte que

- pour tout $(\xi, \varepsilon) \in V \times S_{\theta}^{\prime}$, le point $(\xi, \varphi(\xi, \varepsilon))$ appartient à $U_{0}$;

- $\varphi$ est solution de $\left(\mathcal{E}_{F}\right)$;

- $\varphi$ admet un développement asymptotique $\varphi_{0}+\sum_{n \geq 1} \psi_{n} \varepsilon^{n} \in \mathcal{O}(V)[[\varepsilon]]$ au sens de Poincaré; cela signifie que pour tout secteur $S$ tel que $S \Subset S_{\theta}^{\prime}$ et pour

(1) La notation $S \Subset S_{0}$ signifie que le secteur $S$ est contenu dans $S_{0}$ et que, dans le cercle des directions de $\mathbb{C}$, l'ouverture de $S$ est un arc ouvert simplement connexe et relativement compact dans l'arc ouvert des directions de $S_{0}$.

TOME $128-2000-\mathrm{N}^{\circ} 4$ 
tout $N \in \mathbb{N}^{*}$, il existe une constante réelle positive $C$ de sorte que, pour tout $(\xi, \varepsilon) \in V \times S$, on ait

$$
\left|\varphi(\xi, \varepsilon)-\varphi_{0}(\xi)-\sum_{n=1}^{N-1} \psi_{n}(\xi) \varepsilon^{n}\right| \leq C \varepsilon^{N} .
$$

$S^{\prime}$ 'il existe des solutions $\mathcal{C}$-surstables de l'équation $\left(\mathcal{E}_{F}\right)$ dans toute direction $\theta$ $d u$ secteur $S_{0}$, on dit que $F$ est une équation $\mathcal{C}$-surstable.

Le lecteur averti peut remarquer une différence avec la définition usuelle de la surstabilité : on a remplacé la condition habituelle de convergence par une propriété d'existence de développement asymptotique. Cela est sans importance car il est connu que ces deux conditions sont équivalentes [6]. La formulation choisie ici présente l'avantage de mettre immédiatement en évidence le type d'objet qui est important dans ce texte, à savoir les développements asymptotiques des solutions vus comme des solutions formelles de la même équation. Cela amène à poser la définition suivante.

Définition 2. - Étant donné $F \in \mathcal{A}\left(W_{0}, \varphi_{0}, \chi, p\right)$, on dit qu'une série formelle

$$
\psi=\sum_{n \geq 0} \psi_{n} \varepsilon^{n} \in \mathbb{C}\{\xi\}[[\varepsilon]]
$$

est une solution $\mathcal{C}$-formelle de $\left(\mathcal{E}_{F}\right)$ lorsque

- $\psi_{0}=\varphi_{0}$;

- $\psi$ est une solution formelle de $\left(\mathcal{E}_{F}\right)$, c'est-à-dire

$$
\varepsilon \frac{\partial}{\partial \xi} \psi=\sum_{n \geq 0} F_{n}(\xi, \psi) \varepsilon^{n} .
$$

On peut se douter qu'il y a un lien très fort entre le problème de l'existence de solutions $\mathcal{C}$-surstables et celui de l'existence de solutions $\mathcal{C}$-formelles.

On se donne $F \in \mathcal{A}\left(W_{0}, \varphi_{0}, \chi, p\right)$. Pour étudier l'existence des solutions $\mathcal{C}$ formelles et des solutions $\mathcal{C}$-surstables de $\left(\mathcal{E}_{F}\right)$, il est naturel d'effectuer les changements de variables $x=\xi-\xi_{0}$ et $y=u-\varphi_{0}(\xi)$ qui transforment $\left(\xi_{0}, u_{0}\right)$ en $(0,0)$ et la courbe lente $\mathcal{C}$ en la droite $y=0$ de $\mathbb{C}^{2}$. On obtient ainsi une nouvelle équation

$$
\varepsilon \frac{\mathrm{d} y}{\mathrm{~d} x}=F\left(\xi_{0}+x, \varphi_{0}\left(\xi_{0}+x\right)+y, \varepsilon\right)-\varepsilon \varphi_{0}^{\prime}\left(\xi_{0}+x\right)
$$

qui, compte tenu des hypothèses faites sur $F$, est de la forme $\left(\mathcal{E}_{\phi}^{\prime}\right)$ suivante

$$
\varepsilon \frac{\mathrm{d} y}{\mathrm{~d} x}=x^{p} \chi(x) y+\phi(x, y, \varepsilon)
$$

où $\phi$ est une fonction analytique définie sur un domaine de $\mathbb{C}^{3}$ de la forme $U_{1} \times S_{0}$ telle que : 
- $U_{1}$ est un voisinage ouvert de 0 dans $\mathbb{C}^{2}$ qui est l'image de $U_{0}$ par l'isomorphisme analytique $(\xi, u) \mapsto\left(\xi-\xi_{0}, u-\varphi_{0}(\xi)\right)$;

- $\phi$ admet un développement asymptotique $\sum_{n \geq 0} \phi_{n} \varepsilon^{n}$ Gevrey d'ordre 1 lorsque $\varepsilon$ tend vers 0 (même définition que pour $F$ );

- les fonctions analytiques $x \mapsto \phi_{0}(x, 0)$ et $x \mapsto \frac{\partial}{\partial y} \phi_{0}(x, 0)$ sont nulles au voisinage de 0 .

Enfin, on note $V_{1}$ le voisinage ouvert de 0 dans $\mathbb{C}$ tel que $\xi_{0}+V_{1}=V_{0}$.

$\grave{A}$ travers le changement de variable précédent, une solution $\mathcal{C}$-formelle est une série formelle $f=\sum_{n>1} f_{n} \varepsilon^{n}$ (sans terme en $\varepsilon^{0}$ ) dont les coefficients sont des fonctions holomorphes $f_{n}$ définies au voisinage de 0 dans $\mathbb{C}$, telle que

$$
\varepsilon \frac{\partial}{\partial x} f=x^{p} \chi(x) f+\phi(x, f, \varepsilon) .
$$

En fait, on va montrer qu'il existe de manière unique des séries formelles $f=\sum_{n \geq 1} f_{n} \varepsilon^{n} \in \varepsilon \mathcal{O}\left(V_{1}\right)[[\varepsilon]]$ et $g=\sum_{n>1} g_{n} \varepsilon^{n} \in \varepsilon \mathbb{C}[x]_{p}[[\varepsilon]]$ (autrement dit, les $f_{n}$ sont des fonctions analytiques sur $\bar{V}_{1}$ et les $g_{n}$ sont des polynômes de degré inférieur ou égale à $p-1$ ) telles que

$$
\varepsilon \frac{\partial}{\partial x} f=x^{p} \chi(x) f+\phi(x, f, \varepsilon)+g
$$

Pour cela, on remarque que tout $h \in \varepsilon \mathcal{O}\left(V_{1}\right)[[\varepsilon]]$ s'écrit de manière unique

$$
h=x^{p} \chi(x) f+g
$$

avec $f \in \varepsilon \mathcal{O}\left(V_{1}\right)[[\varepsilon]]$ et $g \in \varepsilon \mathbb{C}[x]_{p}[[\varepsilon]]$. On note

$$
g=\mathcal{T}_{p}(h), \quad f=\mathcal{R}_{p}(h)
$$

ce qui fait que le changement d'inconnue $h=x^{p} \chi(x) f+g$ transforme l'équation (5) en une équation au point fixe dans $\varepsilon \mathcal{O}\left(V_{1}\right)[[\varepsilon]]$

$$
h=\varepsilon \frac{\partial}{\partial x} \mathcal{R}_{p}(h)-\phi\left(x, \mathcal{R}_{p}(h), \varepsilon\right) .
$$

Les opérateurs $\mathcal{T}_{p}$ et $\mathcal{R}_{p}$ ainsi que l'analogue formel de (6) vont jouer un rôle fondamental dans toute la suite de cette étude. Pour l'instant, on se contente de vérifier le lemme suivant.

LEMME 1. - L'équation (6) admet une unique solution dans $\varepsilon \mathbb{C}\{x\}[[\varepsilon]]$ et de plus, cette solution appartient $\grave{a} \varepsilon \mathcal{O}\left(V_{1}\right)[[\varepsilon]]$.

TOME $128-2000-\mathrm{N}^{\circ} 4$ 
Preuve du lemme. - Il est possible de montrer directement qu'une récurrence donne de manière unique les coefficients d'une solution formelle de cette équation. Cependant, pour préparer le lecteur aux techniques exposées dans les paragraphes précédents, il est préférable d'introduire une problématique de point fixe d'opérateur.

Pour tout $h \in \varepsilon \mathcal{O}\left(V_{1}\right)[[\varepsilon]]$, on pose

$$
\mathcal{F}(h)=\varepsilon \frac{\partial}{\partial x} \mathcal{R}_{p}(h)-\phi\left(x, \mathcal{R}_{p}(h), \varepsilon\right)
$$

et on vérifie en combinant le développement asymptotique par rapport à $\varepsilon$ et le développement de Taylor par rapport à la variable $y$ que $\mathcal{F}(h) \in \varepsilon \mathcal{O}\left(V_{1}\right)[[\varepsilon]]$. De plus, pour tout $(h, \widehat{h}) \in \varepsilon \mathcal{O}\left(V_{1}\right)[[\varepsilon]]^{2}$, on a

$$
\mathcal{F}(h+\widehat{h})-\mathcal{F}(h)=\varepsilon \frac{\partial}{\partial x} \mathcal{R}_{p}(\widehat{h})+\mathcal{R}_{p}(\widehat{h}) \frac{\partial}{\partial y} \phi\left(x, \mathcal{R}_{p}(h), \varepsilon\right)\left(\left(\mathcal{R}_{p}(\widehat{h})\right)^{2}\right) .
$$

Puisque $\mathcal{R}_{p}(\widehat{h})$ et $\mathcal{R}_{p}(h)$ appartiennent à $\varepsilon \mathcal{O}\left(V_{1}\right)[[\varepsilon]]$, on obtient en tenant compte des propriétés de $\phi$, les égalités suivantes

$$
\begin{aligned}
\mathcal{F}(h+\widehat{h})-\mathcal{F}(h) & =\varepsilon \frac{\partial}{\partial x} \mathcal{R}_{p}(\widehat{h})+\mathcal{R}_{p}(\widehat{h}) \frac{\partial}{\partial y} \phi_{0}\left(x, \mathcal{R}_{p}(h)\right) \bmod \left(\varepsilon \mathcal{R}_{p}(\widehat{h})\right) \\
& =\varepsilon \frac{\partial}{\partial x} \mathcal{R}_{p}(\widehat{h})+\mathcal{R}_{p}(\widehat{h}) \mathcal{R}_{p}(h) \frac{\partial^{2}}{\partial y^{2}} \phi_{0}(x, 0) \bmod \left(\varepsilon \mathcal{R}_{p}(\widehat{h})\right)
\end{aligned}
$$

ce qui prouve que l'opérateur $\mathcal{F}$ est une contraction formelle au sens suivant

$$
\forall(h, \widehat{h}) \in \varepsilon \mathcal{O}\left(V_{1}\right)[[\varepsilon]], \quad \mathcal{F}(h+\widehat{h})=\mathcal{F}(h) \bmod \left(\varepsilon \frac{\partial}{\partial x} \mathcal{R}_{p}(\widehat{h}), \varepsilon \mathcal{R}_{p}(\widehat{h})\right) .
$$

Il en découle que $\mathcal{F}$ possède au plus un point fixe dans $\varepsilon \mathcal{O}\left(V_{1}\right)[[\varepsilon]]$. On considère alors la suite itérative $\left(f^{n}\right)$ dans $\varepsilon \mathcal{O}\left(V_{1}\right)[[\varepsilon]]$ définie par

$$
f^{0}=0, \quad f^{n+1}=\mathcal{F}\left(f^{n}\right) \text { pour tout } n \in \mathbb{N} .
$$

On vérifie que $f^{n+1}=f^{n}\left[\bmod \left(\varepsilon^{n}\right)\right]$ pour tout $n \in \mathbb{N}$, ce qui fait que cette suite converge formellement dans $\varepsilon \mathcal{O}\left(V_{1}\right)[[\varepsilon]]$ et que sa limite est un point fixe de $\mathcal{F}$.

Cette construction d'un point fixe de $\mathcal{F}$ peut être menée dans l'espace $\varepsilon \mathbb{C}\{x\}[[\varepsilon]]$, ce qui prouve que la solution trouvée est aussi unique dans ce dernier.

Revenant à l'équation initiale, on a démontré le résultat suivant. 
Proposition 1. - Étant donné $F \in \mathcal{A}\left(W_{0}, \varphi_{0}, \chi, p\right)$, il existe de manière unique des séries formelles

$$
f=\varphi_{0}+\sum_{n \geq 1} f_{n} \varepsilon^{n} \in \mathbb{C}\{\xi\}[[\varepsilon]] \quad \text { et } \quad g=\sum_{n \geq 1} g_{n} \varepsilon^{n} \in \varepsilon \mathbb{C}[\xi]_{p}[[\varepsilon]]
$$

telles que

$$
\varepsilon \frac{\partial f}{\partial \xi}=F(\xi, f, \varepsilon)+g
$$

et de plus, $f \in \mathcal{O}\left(V_{0}\right)[[\varepsilon]]$.

En reprenant les notations de cette proposition, la correspondance $F \mapsto g$ définit une application

$$
\mathcal{K}: \mathcal{A}\left(W_{0}, \varphi_{0}, \chi, p\right) \longrightarrow \varepsilon \mathbb{C}[\xi]_{p}[[\varepsilon]]
$$

de sorte que, pour tout $F \in \mathcal{A}\left(W_{0}, \varphi_{0}, \chi, p\right)$, l'équation $\left(\mathcal{E}_{F}\right)$ admet une solution $\mathcal{C}$-formelle si et seulement si $\mathcal{K}(F)=0$.

Dans la suite de ce travail, on se propose de montrer que l'application $\mathcal{K}$ se factorise dans des espaces de séries Gevrey qui sont des limites inductives d'espaces de Banach. De cette manière on obtient que la condition $\mathcal{K}(F)=0$ est aussi une caractérisation de la $\mathcal{C}$-surstabilité. De plus on montre que $\mathcal{K}$ acquiert des propriétés de régularité qui permettent de donner une sorte de description géométrique de l'ensemble des équations $\mathcal{C}$-surstables.

\section{Les algèbres de Banach $E_{\rho}$ et $F_{\rho}$}

L'objet de ce paragraphe est de mettre en place une famille d'espaces de Banach dont les éléments appartiennent à $\mathcal{O}(D(0, R))[[\varepsilon]]$ et sont Gevrey d'ordre 1, espaces dans lesquels seront recherchées les éventuelles solutions formelles surstables pour des équations du type (4). Cela pose le problème de mesurer la taille des coefficients $f_{n}$ pour une série formelle $\sum_{n \geq 1} f_{n} \varepsilon^{n} \in$ $\mathcal{O}(D(0, R))[[\varepsilon]]$. L'idée qui vient immédiatement à l'esprit est d'utiliser la «norme sup 》 sur $D(0, R)$

$$
\|h\|^{(R)}=\sup \{|h(x)| ; x \in D(0, R)\},
$$

mais cette dernière ne permet pas de manipuler agréablement les opérateurs $f \mapsto \varepsilon \partial / \partial x f$ et $f \mapsto(f-f(0)) / x$ qui interviennent de manière fondamentale dans le problème étudié. Comme cela a été fait dans [6], [12], [13], il est préférable d'utiliser une famille dénombrable de normes bien adaptée aux problèmes de perturbations singulières : ce sont les normes de Nagumo.

TOME $128-2000-\mathrm{N}^{\circ} 4$ 
Pour introduire ces dernières, on fixe une fois pour toute des nombres réels $r$ et $R$ tels que $0<r<R$. On a aussi besoin, pour définir un opérateur important pour la suite, de se donner une fonction

$$
\chi: D(0, R) \longrightarrow \mathbb{C}
$$

qui est holomorphe, sans zéro, bornée et telle que la fonction $1 / \chi$ soit aussi bornée sur $D(0, R)$.

\subsection{Les normes de Nagumo.}

On introduit sur le disque ouvert $D(0, R)$ du plan complexe $\mathbb{C}$ une «fonction distance au bord modifiée» définie par

$$
d(x)= \begin{cases}(R-|x|) /(R-r) & \text { si } r<|x|<R \\ 1 & \text { si }|x| \leq r\end{cases}
$$

Afin d'éviter certains problèmes techniques, on a modifié par le facteur $1 /(R-r)$ la fonction proposée dans [6].

On associe à cette fonction $d$ la famille $\left(N_{n}\right)_{n \in \mathbb{N}}$ des «normes de Nagumo» définie pour toute fonction holomorphe $h: D(0, R) \rightarrow \mathbb{C}$ et pour tout $n \in \mathbb{N}$ par

$$
N_{n}(h)=\sup \left\{|h(x)| \cdot d(x)^{n} ; x \in D(0, R)\right\} .
$$

On remarque que $N_{0}$ est égale à la «norme» usuelle \|\|$^{(R)}$ de la convergence uniforme sur $D(0, R)$.

Les normes de Nagumo possèdent des propriétés intéressantes dont voici les plus immédiates (dans l'énoncé desquelles $h$ et $g$ désignent des fonctions holomorphes sur $D(0, R), \alpha$ un nombre complexe et $x$ un élément quelconque de $D(0, R))$ :

$$
\begin{array}{rlrl}
N_{n}(h) & \leq\|h\|^{(R)}, & & |h(x)| \leq N_{n}(h) d(x)^{-n}, \\
N_{n}(h+g) & \leq N_{n}(h)+N_{n}(g), & N_{n}(\alpha h)=|\alpha| \cdot N_{n}(h), \\
N_{n+m}(h g) & \leq N_{n}(h) N_{m}(g) & &
\end{array}
$$

avec quelques exceptions évidentes :

- $\alpha=0$ et $N_{n}(h)=+\infty$ pour la quatrième propriété;

- $\left(h, N_{m}(g)\right)=(0,+\infty)$ ou $\left(g, N_{n}(h)\right)=(0,+\infty)$ pour la cinquième propriété.

La propriété fondamentale des normes de Nagumo, sans équivalent pour la norme \|\|$^{(R)}$ de la convergence uniforme, est relative à la dérivation des fonctions holomorphes. En effet, s'il est possible, pour la norme de la convergence uniforme, de majorer la norme d'une dérivée $h^{\prime}$ en fonction de la norme de $h$, cela se fait à la condition de diminuer le domaine de $h^{\prime}$. La famille des normes de Nagumo est conçue de manière à éliminer cette contrainte de restriction des domaines. 
Lemme 2. - Pour tout $n \in \mathbb{N}$ et pour toute fonction holomorphe $h$ sur $D(0, R)$, si $N_{n}(h)$ est fini, alors il en est de même pour $N_{n+1}(h)$ et

$$
N_{n+1}\left(h^{\prime}\right) \leq \frac{e(n+1)}{R-r} N_{n}(h)
$$

Preuve du lemme. - L'analogue de cette formule est démontré dans [6], [12]. Puisque la fonction $d(x)$ a été légèrement modifiée, on en redonne ici la preuve complète. Pour $x \in D(0, R)$ et $\eta \in] 0, R-|x|[$, la formule de Cauchy donne

$$
h^{\prime}(x)=\frac{1}{2 \pi i} \int_{\partial^{+} D(x, \eta)} \frac{\bullet h(z)}{(z-x)^{2}} \mathrm{~d} z
$$

ce qui fournit la majoration

$$
\left|h^{\prime}(x)\right| \leq \frac{N_{n}(h)}{\eta} \sup _{z \in \partial D(x, \eta)}(d(z))^{-n}
$$

On vérifie sans peine que, pour tout $(z, x) \in D(0, R)$, on a

$$
|d(z)-d(x)| \leq \frac{|z-x|}{R-r}
$$

d'où il vient que $d(z) \geq d(x)-\eta /(R-r)$ pour tout $z \in \partial D(x, R)$. Finalement, on a

$$
\left|h^{\prime}(x)\right| \leq N_{n}(f) \frac{1}{\eta}\left(d(x)-\frac{\eta}{R-r}\right)^{-n} .
$$

Sur l'intervalle $] 0, R-|x|\left[\right.$, la fonction $\eta \mapsto 1 / \eta(d(x)-\eta /(R-r))^{-n}$ admet pour minimum

$$
M=\frac{n+1}{R-r}\left(1-\frac{1}{n+1}\right)^{-n}(d(x))^{-n-1}
$$

Comme $(1-1 /(n+1))^{-n}=(1+1 / n)^{n} \leq \mathrm{e}$, on obtient

$$
\left|h^{\prime}(x)\right| \leq \frac{(n+1) \mathrm{e}}{R-r} N_{n}(h)(d(x))^{-n-1}
$$

TOME $128-2000-\mathrm{N}^{\circ} 4$ 


\subsection{Les espaces $E_{\rho}$ et $F_{\rho}$.}

Définition 3. - Étant donné un nombre réel $\rho>0$ indépendant de $R$, on note $E_{\rho}$ l'ensemble des séries formelles $f=\sum_{n \geq 0} f_{n} \varepsilon^{n} \in \mathbb{C}\{x\}[[\varepsilon]]$ dont chaque coefficient $f_{n}$ est une fonction holomorphe sur le disque ouvert $D(0, R)$ (le même pour tout $n)$ de sorte que les conditions suivantes soient vérifiées:

- $N_{n}\left(f_{n}\right)$ est fini pour chaque $n \geq 0$;

- la série $\sum_{n \geq 0} N_{n}\left(f_{n}\right) \frac{\rho^{n}}{n !}$ est convergente.

$L$ 'ensemble $E_{\rho}$ est un espace vectoriel sur $\mathbb{C}$ que l'on peut munir d'une norme \|\|$_{\rho}$ en posant, pour chaque $f=\sum_{n \geq 0} f_{n} \varepsilon^{n} \in E_{\rho}$,

$$
\|f\|_{\rho}=\sum_{n=0}^{+\infty} N_{n}\left(f_{n}\right) \frac{\rho^{n}}{n !} .
$$

Ces conditions d'appartenance à $E_{\rho}$ constituent une propriété de type Gevrey pour les séries formelles considérées. Si l'on tient à introduire une terminologie, on pourrait dire que $E_{\rho}$ est l'espace des séries formelles qui sont Nagumo-Gevrey d'ordre 1 et de type $1 / \rho$ sur $D(0, R)$.

Proposition 2. - Muni de la norme \|\|$_{\rho}$, l'ensemble $E_{\rho}$ est un espace de Banach.

Preuve de la proposition. - Soit $\left(f^{p}\right)_{p \in \mathbb{N}}$ une suite de Cauchy dans $E_{\rho}$ avec les notations $f^{p}=\sum_{n>0} f_{n}^{p} \varepsilon^{n}$. Il découle de la définition de la norme \|\|$_{\rho}$ que, pour chaque $n \in \mathbb{N}$, la suite de fonctions holomorphes $\left(f_{n}^{p}\right)_{p \in \mathbb{N}}$ converge uniformément sur les compacts de $D(0, R)$ vers une fonction $f_{n}$ définie et holomorphe sur $D(0, R)$. De plus, la suite de fonctions continues $g_{p}: x \mapsto f_{n}^{p}(x) d(x)^{n}$ converge uniformément sur $D(0, R)$ vers une fonction continue $g$ qui est nécessairement de la forme $g: x \mapsto f_{n}(x) d(x)^{n}$.

On considère la série formelle $f=\sum_{n>0} f_{n} \varepsilon^{n} \in \mathbb{C}\{x\}[[\varepsilon]]$ et on se propose de montrer que $f$ appartient à $E_{\rho}$ et que $\lim _{p \rightarrow+\infty}\left\|f-f^{p}\right\|_{\rho}=0$.

Étant donné un nombre réel $\epsilon>0$ arbitraire, il existe un nombre $N \in \mathbb{N}$ tel que, pour chaque $p \geq N$ et $q \geq N$

$$
\sum_{n=0}^{+\infty} \sup _{|x|<R}\left(\left|f_{n}^{q}(x)(d(x))^{n}-f_{n}^{p}(x)(d(x))^{n}\right|\right) \frac{\rho^{n}}{n !}<\epsilon .
$$

Pour chaque nombre $k \in \mathbb{N}$, on obtient

$$
\sum_{n=0}^{k} \sup _{|x|<R}\left(\left|f_{n}^{q}(x)(d(x))^{n}-f_{n}^{p}(x)(d(x))^{n}\right|\right) \frac{\rho^{n}}{n !}<\epsilon .
$$


Fixant $p \geq N$ et faisant tendre $q$ vers $+\infty$, il vient

$$
\sum_{n=0}^{k} \sup _{|x|<R}\left(\left|f_{n}(x)(d(x))^{n}-f_{n}^{p}(x)(d(x))^{n}\right|\right) \frac{\rho^{n}}{n !} \leq \epsilon .
$$

Puisque ceci est vrai pour tout $k \in \mathbb{N}$, on obtient

$$
\sum_{n=0}^{+\infty} \sup _{|x|<R}\left(\left|f_{n}(x)(d(x))^{n}-f_{n}^{p}(x)(d(x))^{n}\right|\right) \frac{\rho^{n}}{n !} \leq \epsilon .
$$

Il en découle que

$$
\sum_{n=0}^{+\infty} N_{n}\left(f_{n}\right) \frac{\rho^{n}}{n !} \leq\left\|f^{p}\right\|_{\rho}+\epsilon<+\infty,
$$

c'est-à-dire que la série formelle $f$ appartient à $E_{\rho}$.

De plus, on a aussi obtenu que, pour chaque $\epsilon>0$, il existe $N \in \mathbb{N}$ tel que, pour tout $p \geq N$, on ait $\left\|f-f^{p}\right\|_{\rho} \leq \epsilon$.

Etant donnés deux éléments $f=\sum_{n \geq 0} f_{n} \varepsilon^{n}$ et $g=\sum_{n \geq 0} g_{n} \varepsilon^{n}$ de $E_{\rho}$, on peut constituer leur produit en tant que série formelle :

$$
f \cdot g=\sum_{n \geq 0}\left(\sum_{k+\ell=n} f_{k} g_{\ell}\right) \varepsilon^{n} .
$$

Proposition 3. - Pour tout $f$ et $g$ dans $E_{\rho}$, la série produit $f \cdot g$ appartient aussi à $E_{\rho}$ et $\|f \cdot g\|_{\rho} \leq\|f\|_{\rho} \cdot\|g\|_{\rho}$.

En d'autres termes, $\left(E_{\rho},\|\|_{\rho}\right)$ est une algèbre de Banach.

Preuve de la proposition. - Elle découle de

$$
N_{n}\left(\sum_{k+\ell=n} f_{k} g_{\ell}\right) \leq \sum_{k+\ell=n} N_{n}\left(f_{k} g_{\ell}\right) \leq \sum_{k+\ell=n} N_{k}\left(f_{k}\right) N_{\ell}\left(g_{\ell}\right)
$$

et de la propriété $1 / n ! \leq 1 / k ! \ell$ ! pour $k+\ell=n$.

Corollaire 1. - Si $f$ est dans $E_{\rho}$ et si g est une fonction holomorphe bornée sur $D(0, R)$, alors le produit $f \cdot g$ appartient à $E_{\rho}$ et $\|f \cdot g\|_{\rho} \leq\|f\|_{\rho} \cdot\|g\|^{(R)}$.

On considère maintenant l'ensemble $F_{\rho}$ des $f=\sum_{n \geq 0} f_{n} \varepsilon^{n} \in E_{\rho}$ dont le terme $f_{0}$ est nul. Il est clair que $F_{\rho}$ est un sous-espace fermé et une sous-algèbre de $E_{\rho}$. Muni de la norme \|\|$_{\rho}$, l'espace $F_{\rho}$ est donc lui-même une algèbre de Banach. 
Il est clair que pour $0<\rho^{\prime}<\rho$, on a les inclusions continues

$$
E_{\rho} \subset E_{\rho^{\prime}} \text { et } \quad F_{\rho} \subset F_{\rho^{\prime}} .
$$

L'ensemble $\mathbb{E}=\bigcup_{\rho>0} E_{\rho}$ pourrait être appelé l'espace des séries formelles Nagumo-Gevrey d'ordre 1 sur $D(0, R)$.

\subsection{Quelques endomorphismes continus de $E_{\rho}$ et $F_{\rho}$.}

Tout endomorphisme $\mathcal{L}$ de l'espace $\mathcal{O}(D(0, R))$ des fonctions holomorphes sur $D(0, R)$ se prolonge aux espaces $E_{\rho}$ et $F_{\rho}$ en posant

$$
\mathcal{L}\left(\sum_{n \geq 0} f_{n} \varepsilon^{n}\right)=\sum_{n \geq 0} \mathcal{L}\left(f_{n}\right) \varepsilon^{n}
$$

ce dernier étant a priori un élément de $\mathcal{O}(D(0, R))[[\varepsilon]]$. Les résultats qui suivent sont énoncés pour $E_{\rho}$ mais ils valent aussi sur l'espace $F_{\rho}$.

C'est le cas de l'opérateur de dérivation $\mathrm{d} / \mathrm{d} x$ sur $\mathcal{O}(D(0, R))$ qui induit l'opérateur de dérivation $\partial / \partial x$ sur $E_{\rho}$ et $F_{\rho}$ défini par

$$
\frac{\partial}{\partial x}\left(\sum_{n \geq 0} f_{n} \varepsilon^{n}\right)=\sum_{n \geq 0} \frac{\mathrm{d} f_{n}}{\mathrm{~d} x} \varepsilon^{n}
$$

Proposition 4. - Pour tout $f \in E_{\rho}$, la série formelle $\varepsilon \partial / \partial x f$ appartient $\grave{a} E_{\rho}$ et on $a$

$$
\left\|\varepsilon \frac{\partial}{\partial x} f\right\|_{\rho} \leq \frac{\mathrm{e} \rho}{R-r}\|f\|_{\rho}
$$

Autrement dit, l'opérateur $f \mapsto \varepsilon \partial / \partial x f$ est un endomorphisme continu de $E_{\rho}$ dont la norme est inférieure ou égale à e $\rho /(R-r)$ (où e désigne le nombre réel dont le logarithme népérien vaut 1).

Preuve de la proposition. - C'est une conséquence directe de la propriété fondamentale des normes de Nagumo énoncé dans le lemme 2. En effet, de ce lemme, on déduit

$$
\left\|\varepsilon \frac{\partial}{\partial x} f\right\|_{\rho} \leq \frac{\mathrm{e} \rho}{R-r} \sum_{n=0}^{+\infty}(n+1) N_{n}\left(f_{n}\right) \frac{\rho^{n+1}}{(n+1) !}=\frac{\mathrm{e} \rho}{R-r}\|f\|_{\rho} .
$$

On considère maintenant l'opérateur de décalage $\mathcal{S}$ qui, à une fonction holomorphe $h$ définie sur un voisinage $O$ de 0 dans $\mathbb{C}$, fait correspondre la fonction holomorphe $\mathcal{S} h$ sur $O$ telle que, pour tout $x \in O$,

$$
h(x)=h(0)+x \mathcal{S h}(x) .
$$


Cet opérateur se prolonge à $E_{\rho}$ en posant, pour $f=\sum_{n \geq 1} f_{n} \varepsilon^{n}$

$$
\mathcal{S} f=\sum_{n \geq 1} \mathcal{S} f_{n} \varepsilon^{n}
$$

Proposition 5. - Pour tout $f \in E_{\rho}$, la série formelle $\mathcal{S} f$ appartient à $E_{\rho}$ et on $a\|\mathcal{S} f\|_{\rho} \leq 2 / r\|f\|_{\rho}$.

Autrement dit, l'opérateur $\mathcal{S}$ est un endomorphisme continu de $E_{\rho}$ dont la norme est inférieure ou égale à $2 / r$. Ce résultat découle de la propriété analogue suivante des normes de Nagumo [6].

Lemme 3. - Pour tout $n \in \mathbb{N}$ et pour toute fonction holomorphe $h$ sur $D(0, R)$, si $N_{n}(h)$ est fini, alors il en est de même pour $N_{n}(\mathcal{S} h)$ et $N_{n}(\mathcal{S} h) \leq 2 / r N_{n}(h)$.

Preuve du lemme. - Soit $x \in D(0, R)$.

- Si $r \leq|x| \leq R$, on a

$$
|\mathcal{S} h(x)| \leq \frac{|h(x)|+|h(0)|}{x} \leq \frac{(d(x))^{-n} N_{n}(h)+N_{n}(h)}{r} \leq \frac{2}{r} N_{n}(h)(d(x))^{-n}
$$

car $d(0)=1$ et $d(x) \leq 1$.

- Si au contraire $|x| \leq r$, on a

$$
|\mathcal{S} h(x)| \leq \sup _{|u|=r}|\mathcal{S} h(u)| \leq \frac{2}{r} N_{n}(h)
$$

d'après le cas précédent, ce qui donne le résultat car $d(x)=1$.

Pour tout nombre $p \in \mathbb{N}$, l'application de l'opérateur $\mathcal{S}^{p} \operatorname{sur} \mathcal{O}(D(0, R))$ consiste à soustraire le polynôme de Taylor d'ordre $p-1$ en 0 puis à diviser le résultat de la soustraction par $x^{p}$.

$$
\forall h \in \mathcal{O}(D(0, R)), \forall x \in D(0, R), \quad h(x)=\sum_{k=0}^{p-1} \frac{h^{(k)}(0)}{k !} x^{k}+x^{p} \mathcal{S}^{p}(h)(x) .
$$

Soit $\mathcal{T}_{p}$ l'application qui, à une fonction holomorphe $h$ définie sur un voisinage de 0 dans $\mathbb{C}$, fait correspondre son polynôme de Taylor d'ordre $p-1$ en 0 , c'està-dire

$$
\mathcal{T}_{p}(h)=\sum_{k=0}^{p-1} \frac{h^{(k)}(0)}{k !} x^{k}
$$

Cette application se prolonge à $E_{\rho}$ en un opérateur encore noté $\mathcal{T}_{p}$.

TOME $128-2000-\mathrm{N}^{\circ} 4$ 
Proposition 6. - L'opérateur $\mathcal{T}_{p}$ est un endomorphisme continu de $E_{\rho}$.

Preuve de la proposition. - Cela découle de la décomposition $\mathcal{T}_{p}=\mathrm{id}-x^{p} \mathcal{S}^{p}$ et de l'inégalité

$$
\left\|x^{p} \mathcal{S}^{p}(f)\right\|_{\rho} \leq R^{p}\left(\frac{2}{r}\right)^{p}\|f\|_{\rho} .
$$

Nous avons aussi besoin de l'opérateur $\mathcal{R}_{p}=1 / \chi \mathcal{S}^{p}$ où $\chi$ désigne la fonction holomorphe précisée au début du paragraphe 3 . Le résultat qui suit est immédiat.

Proposition 7. - Pour tout $f \in E_{\rho}$, la série formelle $\mathcal{R}_{p} f$ appartient à $E_{\rho}$ et on a

$$
\left\|\mathcal{R}_{p} f\right\|_{\rho} \leq\left(\frac{2}{r}\right)^{p}\left\|\frac{1}{\chi}\right\|^{(R)}\|f\|_{\rho} .
$$

On rappelle que $\|1 / \chi\|^{(R)}$ désigne la norme de la convergence uniforme de la fonction $1 / \chi$ sur le disque $D(0, R)$.

\section{Un opérateur de substitution dans $F_{\rho}$}

On fixe une fois pour toute un nombre réel $R^{\prime}$ strictement positif indépendant de $r, R$ et $\rho$.

On considère maintenant une série formelle

$$
\phi=\sum_{n \geq 0} \phi_{n}(x, y) \varepsilon^{n} \in \mathbb{C}\{x, y\}[[\varepsilon]] .
$$

Étant donné $f=\sum_{n \geq 1} f_{n}(x) \varepsilon^{n} \in F_{\rho}$, on peut constituer la série formelle $\widetilde{\phi}(f) \in \mathbb{C}\{x\}[[\varepsilon]]$ obtenue en substituant $f$ à l'indéterminée $y$ dans l'expression de $\phi$

$$
\widetilde{\phi}(f)=\sum_{n \geq 0} \phi_{n}(x, f) \varepsilon^{n} .
$$

Afin de s'interroger sur l'appartenance éventuelle de $\widetilde{\phi}(f)$ à $F_{\rho}$, on considère les conditions suivantes susceptibles d'être satisfaites par $\phi$ :

$\left(\mathrm{H}_{1}\right)$ les coefficients $\phi_{n}$ sont des fonctions analytiques bornées

$$
\begin{aligned}
\phi_{n}: D(0, R) \times D\left(0, R^{\prime}\right) & \longrightarrow \mathbb{C}, \\
(x, y) & \longmapsto \phi_{n}(x, y)
\end{aligned}
$$

( $R$ et $R^{\prime}$ étant déjà fixés sont donc indépendants de $n$ );

$\left(\mathrm{H}_{2}\right)$ la série $\phi$ est Gevrey au sens où $\sum_{n=0}^{+\infty}\left\|\phi_{n}\right\|^{\left(R, R^{\prime}\right)} \rho^{n} / n !<+\infty$ (expression dans laquelle \|\|$^{\left(R, R^{\prime}\right)}$ désigne la norme de la convergence uniforme sur $\left.D(0, R) \times D\left(0, R^{\prime}\right)\right)$;

$\left(\mathrm{H}_{3}\right)$ la fonction $x \mapsto \phi_{0}(x, 0)$ est nulle. 
Proposition 8. - Sous les hypothèses $\left(\mathrm{H}_{1}\right)$ et $\left(\mathrm{H}_{2}\right)$, pour tout $f \in F_{\rho}$ tel que $\|f\|_{\rho}<R^{\prime}$, la série formelle $\widetilde{\phi}(f)$ appartient à $E_{\rho}$ et

$$
\|\widetilde{\phi}(f)\|_{\rho} \leq \frac{R^{\prime}}{R^{\prime}-\|f\|_{\rho}} \sum_{n=0}^{+\infty}\left\|\phi_{n}\right\|^{\left(R, R^{\prime}\right)} \frac{\rho^{n}}{n !} .
$$

Si de plus la condition $\left(\mathrm{H}_{3}\right)$ est satisfaite, la série formelle $\widetilde{\phi}(f)$ appartient à $F_{\rho}$ et

$$
\|\widetilde{\phi}(f)\|_{\rho} \leq \frac{R^{\prime}}{R^{\prime}-\|f\|_{\rho}}\left(\frac{\|f\|_{\rho}}{R^{\prime}}\left\|\phi_{0}\right\|^{\left(R, R^{\prime}\right)}+\sum_{n=1}^{+\infty}\left\|\phi_{n}\right\|^{\left(R, R^{\prime}\right)} \frac{\rho^{n}}{n !}\right) .
$$

Preuve de la proposition 8. - Pour chaque $n \in \mathbb{N}$, la fonction $\phi_{n}$ est la somme d'une série convergente

$$
\forall(x, y) \in D(0, R) \times D\left(0, R^{\prime}\right), \quad \phi_{n}(x, y)=\sum_{k=0}^{+\infty} \phi_{n, k}(x) y^{k}
$$

où chaque $\phi_{n, k}$ est analytique sur $D(0, R)$. D'après la formule de Cauchy,

$$
\left\|\phi_{n, k}\right\|^{(R)} \leq \frac{\left\|\phi_{n}\right\|^{\left(R, R^{\prime}\right)}}{R^{\prime k}}
$$

En utilisant les propriétés de la norme \|\|$_{\rho}$ il vient

$$
\left\|\phi_{n, k} f^{k}\right\|_{\rho} \leq\left\|\phi_{n, k}\right\|_{\rho}\left(\|f\|_{\rho}\right)^{k}=\left\|\phi_{n, k}\right\|^{(R)}\left(\|f\|_{\rho}\right)^{k} \leq\left\|\phi_{n}\right\|^{\left(R, R^{\prime}\right)}\left(\frac{\|f\|_{\rho}}{R^{\prime}}\right)^{k}
$$

On en déduit que chaque $\phi_{n, k} f^{k}$ appartient à $E_{\rho}$. De plus, pour $\|f\|_{\rho}<R^{\prime}$, la série de terme général $u_{k}=\phi_{n, k} f^{k}$ converge dans $E_{\rho}$ et

$$
\left\|\sum_{k=0}^{\infty} \iota_{k}\right\|_{\rho} \leq\left\|\phi_{n}\right\|^{\left(R, R^{\prime}\right)} \frac{R^{\prime}}{R^{\prime}-\|f\|_{\rho}}
$$

Du fait que, pour tout $N \in \mathbb{N}$, le coefficient de $\varepsilon^{N}$ dans la série formelle $\widetilde{\phi_{n}}(f)=\sum_{k \geq 0} \phi_{n, k} f^{k}$ provient de la somme partielle $\sum_{k=0}^{N} \phi_{n, k} f^{k}$, on a $\sum_{k=0}^{\infty} u_{k}=\widetilde{\phi_{n}}(f)$, d'où il découle que $\widetilde{\phi_{n}}(f)$ appartient à $E_{\rho}$ et que

$$
\left\|\widetilde{\phi_{n}}(f)\right\|_{\rho} \leq\left\|\phi_{n}\right\|^{\left(R, R^{\prime}\right)} \frac{R^{\prime}}{R^{\prime}-\|f\|_{\rho}} .
$$

TOME $128-2000-\mathrm{N}^{\circ} 4$ 
On obtient que

$$
\left\|\widetilde{\phi_{n}}(f) \varepsilon^{n}\right\|_{\rho} \leq\left\|\phi_{n}\right\|^{\left(R, R^{\prime}\right)} \frac{R^{\prime}}{R^{\prime}-\|f\|_{\rho}} \frac{\rho^{n}}{n !}
$$

ce qui montre que la série de terme général $\widetilde{\phi_{n}}(f) \varepsilon^{n}$ converge dans $E_{\rho}$ et sa somme est égale à la série formelle $\widetilde{\phi}(f)=\sum_{n \geq 0} \widetilde{\phi}_{n}(f) \varepsilon^{n}$. Cette dernière appartient donc à $F_{\rho}$ et

$$
\|\widetilde{\phi}(f)\|_{\rho} \leq \frac{R^{\prime}}{R^{\prime}-\|f\|_{\rho}} \sum_{n=0}^{+\infty}\left\|\phi_{n}\right\|^{\left(R, R^{\prime}\right)} \frac{\rho^{n}}{n !}
$$

Si la fonction $x \mapsto \phi_{0}(x, 0)$ est nulle, alors la série formelle $\widetilde{\phi}(f)$ admet 0 comme terme en $\varepsilon^{0}$, et donc elle appartient à $F_{\rho}$. De plus, dans ce cas on a $\widetilde{\phi_{0}}(f)=\sum_{k \geq 1} \phi_{0, k} f^{k}$ d'où la majoration

$$
\left\|\widetilde{\phi_{0}}(f)\right\|_{\rho} \leq\left\|\phi_{0}\right\|^{\left(R, R^{\prime}\right)} \frac{\|f\|_{\rho}}{R^{\prime}-\|f\|_{\rho}}
$$

D'après la proposition précédente et sous les hypothèses $\left(\mathrm{H}_{i}\right), i=1,2,3$, l'opérateur $\widetilde{\phi}$ est une application à valeurs dans $F_{\rho}$ et définie sur la boule ouverte $B_{F_{\rho}}\left(0, R^{\prime}\right)$ de centre 0 et de rayon $R^{\prime}$ de l'espace de Banach $F_{\rho}$. On s'interroge maintenant sur l'éventuelle différentiabilité de $\widetilde{\phi}$.

Pour cela, on fait l'hypothèse supplémentaire suivante :

$\left(\mathrm{H}_{4}\right)$ les séries dérivées $\partial \phi / \partial y$ et $\partial^{2} \phi / \partial y^{2}$ sont Gevrey au même sens que $\phi$, c'est-à-dire sont telles que

$$
\sum_{n=0}^{+\infty}\left\|\frac{\partial \phi_{n}}{\partial y}\right\|^{\left(R, R^{\prime}\right)} \frac{\rho^{n}}{n !}<+\infty \quad \text { et } \sum_{n=0}^{+\infty}\left\|\frac{\partial^{2} \phi_{n}}{\partial y^{2}}\right\|^{\left(R, R^{\prime}\right)} \frac{\rho^{n}}{n !}<+\infty
$$

Remarquons que, conséquence des inégalités de Cauchy, $\left(\mathrm{H}_{4}\right)$ est satisfaite si $\phi$ est Gevrey sur un domaine un peu plus grand, c'est-à-dire s'il existe $R^{\prime \prime}>R^{\prime}$ tel que chaque $\phi_{n}$ soit analytique bornée sur $D(0, R) \times D\left(0, R^{\prime \prime}\right)$ et vérifie

$$
\sum_{n=0}^{+\infty}\left\|\phi_{n}\right\|^{\left(R, R^{\prime \prime}\right)} \frac{\rho^{n}}{n !}<+\infty
$$


Proposition 9. - Sous les hypothèses $\left(\mathrm{H}_{i}\right) i=1,2,3,4$, l'application $\widetilde{\phi}$ est différentiable de classe $C^{1}$ sur la boule $B_{F_{\rho}}\left(0, R^{\prime}\right)$ de l'espace de Banach $F_{\rho}$ et, pour tout $f$ dans $B_{F_{\rho}}\left(0, R^{\prime}\right)$, la différentielle de $\widetilde{\phi}$ en $f$ est l'application linéaire

$$
D(\widetilde{\phi})(f): g \longmapsto \frac{\widetilde{\partial}}{\partial y} \phi(f) g .
$$

Preuve de la proposition 9. - Soit $f$ appartenant à la boule $B_{F_{\rho}}\left(0, R^{\prime}\right)$ de l'espace $F_{\rho}$. La formule de Taylor donne, pour tout $g \in F_{\rho}$

$$
\widetilde{\phi}(f+g)-\widetilde{\phi}(f)=\widetilde{\frac{\partial}{\partial y}} \phi(f) g+g^{2} \int_{0}^{1}(1-t) \frac{\widetilde{\partial^{2}}}{\partial y^{2}} \phi(f+t g) \mathrm{d} t .
$$

D'après la proposition précédente, $\widetilde{\partial / \partial y} \phi(f)$ appartient à l'espace $E_{\rho}$. Puisque ce dernier est une algèbre de Banach, l'application linéaire $g \mapsto \partial \widetilde{\partial y y} \phi(f) g$ est continue de $F_{\rho}$ dans $F_{\rho}$ et sa norme est inférieure ou égale à $\|\widehat{\partial / \partial y} \phi(f)\|_{\rho}$.

Pour montrer que $\widetilde{\phi}$ est différentiable en $f$, il reste à vérifier que

$$
\left\|\int_{0}^{1}(1-t) \frac{\widetilde{\partial^{2}}}{\partial y^{2}} \phi(f+t g) \mathrm{d} t\right\|_{\rho}
$$

est borné lorsque $g \rightarrow 0$ dans $F_{\rho}$.

De l'écriture en série formelle $\partial^{2} \phi / \partial y^{2}=\sum_{n \geq 0} \psi_{n} \varepsilon^{n}$ où $\psi_{n}=\partial^{2} \phi_{n} / \partial y^{2}$, on déduit que

$$
\int_{0}^{1}(1-t) \frac{\widetilde{\partial^{2}}}{\partial y^{2}} \phi(f+t g) \mathrm{d} t=\sum_{n \geq 0}\left(\int_{0}^{1}(1-t) \widetilde{\psi_{n}}(f+t g) \mathrm{d} t\right) \varepsilon^{n}
$$

Comme précédemment, on a un développement en série convergente

$$
\forall(x, y) \in D(0, R) \times D\left(0, R^{\prime}\right), \quad \psi_{n}(x, y)=\sum_{k=0}^{+\infty} \psi_{n, k}(x) y^{k}
$$

où chaque $\psi_{n, k}$ est analytique sur $D(0, R)$ et borné par $\left\|\psi_{n}\right\|^{\left(R, R^{\prime}\right)} / R^{\prime}$. Pour tout $x \in D(0, R)$ on a le développement

$$
\int_{0}^{1}(1-t) \psi_{n}(x, f+t g) \mathrm{d} t=\sum_{k \geq 0} \psi_{n, k}(x) \int_{0}^{1}(1-t)(f+t g)^{k} \mathrm{~d} t .
$$

TOME $128-2000-\mathrm{N}^{\circ} 4$ 
Puisque $\left\|\int_{0}^{1}(1-t)(f+t g)^{k} \mathrm{~d} t\right\|_{\rho} \leq\left(\|f\|_{\rho}+\|g\|_{\rho}\right)^{k}$, on obtient que

$$
\left\|\psi_{n, k} \int_{0}^{1}(1-t)(f+t g)^{k} \mathrm{~d} t\right\|_{\rho} \leq\left\|\psi_{n}\right\|^{\left(R, R^{\prime}\right)}\left(\frac{\|f\|_{\rho}+\|g\|_{\rho}}{R^{\prime}}\right)^{k}
$$

ce qui prouve que la série de terme général $v_{k}=\psi_{n, k} \int_{0}^{1}(1-t)(f+t g)^{k} \mathrm{~d} t$ converge dans $E_{\rho}$ pour $\|g\|_{\rho}$ assez petit et que sa somme est égale à $\int_{0}^{1}(1-t) \widetilde{\psi_{n}}(f+t g) \mathrm{d} t$ avec la majoration

$$
\left\|\int_{0}^{1}(1-t) \widetilde{\psi_{n}}(f+t g) \mathrm{d} t\right\|_{\rho} \leq\left\|\psi_{n}\right\|^{\left(R, R^{\prime}\right)} \frac{R^{\prime}}{R^{\prime}-\|f\|_{\rho}-\|g\|_{\rho}} .
$$

Finalement, on voit que la série de terme général $\left(\int_{0}^{1}(1-t) \widetilde{\psi_{n}}(f+t g) \mathrm{d} t\right) \varepsilon^{n}$ converge dans $E_{\rho}$ et que sa somme est égale à $\int_{0}^{1}(1-t) \partial^{2} \widetilde{/ \partial y^{2}} \phi(f+t g) \mathrm{d} t$ avec la majoration

$$
\left\|\int_{0}^{1}(1-t) \frac{\widetilde{\partial^{2}}}{\partial y^{2}} \phi(f+t g) \mathrm{d} t\right\|_{\rho} \leq \frac{R^{\prime}}{R^{\prime}-\|f\|_{\rho}-\|g\|_{\rho}} \sum_{n=0}^{+\infty}\left\|\frac{\partial^{2} \phi_{n}}{\partial y^{2}}\right\|^{\left(R, R^{\prime}\right)} \frac{\rho^{n}}{n !} .
$$

Il est maintenant démontré que $\widetilde{\phi}$ est différentiable en $f$, de différentielle égale à $g \mapsto \partial \widehat{/ \partial y} \phi(f) g$.

Pour montrer que $\widetilde{\phi}$ est de classe $C^{1}$, on s'intéresse maintenant à

$$
\| D\left(\widetilde{\phi}(f+h)-D\left(\widetilde{\phi}(f) \|_{\rho}\right.\right.
$$

lorsque $h$ est assez petit dans $F_{\rho}$. Puisque

$$
(D(\widetilde{\phi})(f+h)-D(\widetilde{\phi})(f)) \cdot g=\left(\widetilde{\frac{\partial}{\partial y}} \phi(f+h)-\widetilde{\partial} \partial(f)\right) g
$$

on a

$$
\| D\left(\widetilde{\phi}(f+h)-D\left(\widetilde{\phi}(f)\left\|_{\rho} \leq\right\| \widetilde{\frac{\partial}{\partial y}} \phi(f+h)-\widetilde{\frac{\partial}{\partial y}} \phi(f) \|_{\rho} .\right.\right.
$$

Comme $\partial \widetilde{/ \partial y} \phi(f+h)-\partial \widetilde{/ \partial y} \phi(f)=h \int_{0}^{1} \partial^{2} \widetilde{/ \partial y^{2}} \phi(f+t h) \mathrm{d} t$, il suffit, pour vérifier que $D(\widetilde{\phi})$ est continue en $f$, de montrer que $\left\|\int_{0}^{1} \partial^{2} \widetilde{/ \partial y^{2}} \phi(f+t h) \mathrm{d} t\right\|_{\rho}$ est défini et borné lorsque $h \rightarrow 0$ dans $F_{\rho}$. Cela s'obtient par un calcul analogue au précédent qui donne la majoration

$$
\left\|\int_{0}^{1} \frac{\widetilde{\partial^{2}}}{\partial y^{2}} \phi(f+t h) \mathrm{d} t\right\|_{\rho} \leq \frac{R^{\prime}}{R^{\prime}-\|f\|_{\rho}-\|h\|_{\rho}} \sum_{n=0}^{+\infty}\left\|\frac{\partial^{2} \phi_{n}}{\partial y^{2}}\right\|^{\left(R, R^{\prime}\right)} \frac{\rho^{n}}{n !} .
$$




\section{Un théorème de point fixe dans $\boldsymbol{F}_{\rho}$}

Comme dans le paragraphe précédent, on se donne une série formelle

$$
\phi=\sum_{n \geq 0} \phi_{n} \varepsilon^{n} \in \mathbb{C}\{x, y\}[[\varepsilon]]
$$

dont les coefficients sont des fonctions analytiques $\phi_{n}$ définies et bornées sur $D(0, R) \times D\left(0, R^{\prime}\right)$ satisfaisant les hypothèses $\left(\mathrm{H}_{i}\right)$ pour $i=1,2,3,4$. De plus, on impose à $\phi$ la condition suivante :

$\left(\mathrm{H}_{5}\right)$ La fonction $x \mapsto \partial / \partial y \phi_{0}(x, 0)$ est nulle.

On considère l'application $\mathcal{F}$ définie au voisinage de 0 dans l'espace $F_{\rho}$ par

$$
\mathcal{F}(f)=\varepsilon \frac{\partial}{\partial x} \mathcal{R}_{p} f-\widetilde{\phi}\left(\mathcal{R}_{p} f\right)
$$

où $\mathcal{R}_{p}$ est l'opérateur linéaire continu introduit à la fin du paragraphe 3.3 tel que, pour tout $f \in F_{\rho}$, on ait

$$
f=\sum_{k=0}^{p-1} \frac{x^{k}}{k !} \frac{\partial^{k}}{\partial x^{k}} f_{\left.\right|_{x=0}}+x^{p} \chi(x) \mathcal{R}_{p} f .
$$

Puisque la norme de l'opérateur $\mathcal{R}_{p}$ est majorée par $K_{p}=(2 / r)^{p}\|1 / \chi\|^{(R)}$, l'application $\mathcal{F}$ est définie sur la boule $B_{F_{\rho}}\left(0, K_{p}^{-1} R^{\prime}\right)$ de l'espace de Banach $F_{\rho}$ et elle est à valeurs dans $F_{\rho}$. Plus généralement, pour tout $\rho^{\prime}$ tel que $0<\rho^{\prime} \leq \rho$, l'application $\mathcal{F}$ est définie et différentiable sur la boule $B_{F_{\rho^{\prime}}}\left(0, K_{p}^{-1} R^{\prime}\right)$ de l'espace de Banach $F_{\rho^{\prime}}$ et elle est à valeurs dans $F_{\rho^{\prime}}$.

Lemme 4. - Pour tout $\alpha \in] 0,1[$, il existe $\eta \in] 0, K_{p}^{-1} R^{\prime}\left[\right.$ et $\left.\left.\rho_{0} \in\right] 0, \rho\right]$ tel que, si $\left.\left.\rho^{\prime} \in\right] 0, \rho_{0}\right]$ alors $\|D(\mathcal{F})(f)\|_{\rho^{\prime}} \leq \alpha$ pour tout $f \in B_{F_{\rho^{\prime}}}(0, \eta)$.

Preuve du lemme 4. - Pour tout $\left.\left.\rho^{\prime} \in\right] 0, \rho\right], \mathcal{F}$ est différentiable en $f \in$ $B_{F_{\rho^{\prime}}}\left(0, K_{p}^{-1} R^{\prime}\right)$ dans l'espace $F_{\rho^{\prime}}$ et sa différentielle est

$$
D(\mathcal{F})(f): g \longmapsto \varepsilon \frac{\partial}{\partial x} \mathcal{R}_{p} g-\widetilde{\partial} \frac{\widetilde{\partial}}{\partial y} \phi\left(\mathcal{R}_{p} f\right) \mathcal{R}_{p} g
$$

On en déduit la majoration suivante

$$
\begin{aligned}
\|D(\mathcal{F})(f)\|_{\rho^{\prime}} \leq \frac{K_{p} \mathrm{e} \rho^{\prime}}{R-r}+\frac{K_{p} R^{\prime}}{R^{\prime}-K_{p}\|f\|_{\rho^{\prime}}}\left(\frac{K_{p}\|f\|_{\rho^{\prime}}}{R^{\prime}}\left\|\frac{\partial}{\partial y} \phi_{0}\right\|^{\left(R, R^{\prime}\right)}\right. & \\
& \left.+\sum_{n=1}^{+\infty}\left\|\frac{\partial}{\partial y} \phi_{n}\right\|^{\left(R, R^{\prime}\right)} \frac{\rho^{\prime n}}{n !}\right) .
\end{aligned}
$$

TOME $128-2000-\mathrm{N}^{\circ} 4$ 
Soit $\eta$ un nombre réel tel que $0<\eta<K_{p}^{-1} R^{\prime}$ de sorte que, pour $\|f\|_{\rho^{\prime}}<\eta$, on ait

$$
\frac{K_{p}^{2}\|f\|_{\rho^{\prime}}}{R^{\prime}-K_{p}\|f\|_{\rho^{\prime}}}\left\|\frac{\partial}{\partial y} \phi_{0}\right\|^{\left(R, R^{\prime}\right)}<\frac{\alpha}{2} .
$$

Maintenant, il existe $\rho_{0}>0$ de manière à ce que, pour $\rho^{\prime}<\rho_{0}$ et pour $\|f\|_{\rho^{\prime}}<\eta$, on ait

$$
K_{p} \frac{\mathrm{e} \rho^{\prime}}{R-r}+K_{p} \frac{R^{\prime}}{R^{\prime}-K_{p}\|f\|_{\rho^{\prime}}} \sum_{n=1}^{+\infty}\left\|\frac{\partial}{\partial y} \phi_{n}\right\|^{\left(R, R^{\prime}\right)} \frac{\rho^{\prime n}}{n !}<\frac{\alpha}{2}
$$

Pour ce choix de $\rho_{0}$ et de $\eta$, on obtient $\|D(\mathcal{F})(f)\|_{\rho^{\prime}} \leq \alpha$ pour tout $f \in B_{F_{\rho^{\prime}}}(0, \eta)$.

THÉORÈmE 1. - Sous les hypothèses $\left(\mathrm{H}_{i}\right), i=1,2,3,4,5$, il existe $\left.\left.\rho_{1} \in\right] 0, \rho\right]$ tel que l'application $\mathcal{F}$ admette un unique point fixe dans l'espace $F_{\rho^{\prime}}$ pour tout $\left.\left.\rho^{\prime} \in\right] 0, \rho_{1}\right]$.

Bien entendu, il découle du théorème et de l'emboitement des espaces $F_{\rho}$ que l'application $\mathcal{F}$ admet un unique point fixe dans $\bigcup_{\left.\left.\rho^{\prime} \in\right] 0, \rho_{1}\right]} F_{\rho^{\prime}}$.

Preuve du théorème 1 . - On choisit de manière arbitraire $\alpha \in] 0,1[$. Le lemme 4 fournit $\left.\left.\rho_{0} \in\right] 0, \rho\right]$ et $\eta>0$ tel que, pour tout $\left.\left.\rho^{\prime} \in\right] 0, \rho_{0}\right]$, la restriction de $\mathcal{F}$ à la boule $B_{F_{\rho^{\prime}}}(0, \eta)$ est contractante avec constante de contraction égale à $\alpha$.

On pose $f^{0}=0$ puis $f^{1}=\mathcal{F}(0)$ ce qui donne $f^{1}=-\widetilde{\phi}(0)$. Alors, du fait que $\phi_{0}(., 0)=0$,

$$
\left\|f^{1}-f^{0}\right\|_{\rho^{\prime}}=\|\widetilde{\phi}(0)\|_{\rho^{\prime}}=\sum_{n=1}^{+\infty} N_{n}\left(\phi_{n}(., 0)\right) \frac{\rho^{\prime^{n}}}{n !}
$$

On peut donc choisir $\rho_{1}<\rho_{0}$ pour que $\left\|f^{1}-f^{0}\right\|_{\rho^{\prime}}<\frac{1}{2}(1-\alpha) \eta$ lorsque $\rho^{\prime}<\rho_{1}$. Sous cette condition, la suite des itérés $f^{n+1}=\mathcal{F}\left(f^{n}\right)$ converge vers un point fixe de $\mathcal{F}$ dans la boule fermée $\bar{B}\left(0, \frac{1}{2} \eta\right)$ de $F_{\rho^{\prime}}$ et ce point fixe est unique dans cette boule.

Enfin, en procédant comme dans le lemme 1, on vérifie que l'équation $f=\varepsilon \partial / \partial x \mathcal{R}_{p} f-\widetilde{\phi}\left(\mathcal{R}_{p} f\right)$ admet une unique solution dans $\mathbb{C}\{x\}[[\varepsilon]]$. 


\section{Les espaces de Banach $\boldsymbol{G}_{\rho}$ et $\boldsymbol{H}_{\rho}^{p}$}

Les algèbres $F_{\rho}$ introduites précédemment sont les espaces dans lesquels nous allons rechercher les solutions de certaines équations différentielles. Ces dernières vont correspondre aux éléments de nouveaux «espaces fonctionnels formels» obtenus en sélectionnant les conditions sur les fonctions $\phi$ qui ont permis de définir un opérateur de substitution suffisamment régulier et d'obtenir un théorème de point fixe (théorème 1). Pour définir ces espaces, nous avons besoin de la notation suivante : pour $\psi=\sum_{n \geq 0} \psi_{n} \varepsilon^{n}$ appartenant à $\mathcal{O}(D(0, R) \times$ $D\left(0, R^{\prime}\right)[[\varepsilon]]$, on pose, lorsque cela a du sens,

$$
\|\psi\|_{\rho}^{\left(R, R^{\prime}\right)}=\sum_{n=0}^{+\infty}\left\|\psi_{n}\right\|^{\left(R, R^{\prime}\right)} \frac{\rho^{n}}{n !}
$$

où \|\|$^{\left(R, R^{\prime}\right)}$ désigne la norme de la convergence uniforme sur $\left(D(0, R) \times D\left(0, R^{\prime}\right)\right.$.

DÉfinition 4. - Étant donné un nombre réel $\rho$ tel que $\rho>0$, on note $G_{\rho}$ l'ensemble des séries formelles $\phi=\sum_{n \geq 0} \phi_{n} \varepsilon^{n} \in \mathbb{C}\{x, y\}[[\varepsilon]]$ telles que:

- les coefficients $\phi_{n}$ sont des fonctions analytiques $D(0, R) \times D\left(0, R^{\prime}\right) \rightarrow \mathbb{C}$ avec $R$ et $R^{\prime}$ fixés comme précédemment;

- les fonctions $x \mapsto \phi_{0}(x, 0)$ et $x \mapsto \partial / \partial y \phi_{0}(x, 0)$ sont nulles;

- pour tout $n \in \mathbb{N}$, les fonctions $\phi_{n}, \partial / \partial y \phi_{n}$ et $\partial^{2} / \partial y^{2} \phi_{n}$ sont bornées sur $D(0, R) \times D\left(0, R^{\prime}\right)$

- $\|\phi\|_{\rho}^{\left(R, R^{\prime}\right)}<+\infty,\|\partial / \partial y \phi\|_{\rho}^{\left(R, R^{\prime}\right)}<+\infty$ et $\left\|\partial^{2} / \partial y^{2} \phi\right\|_{\rho}^{\left(R, R^{\prime}\right)}<+\infty$.

$L$ 'ensemble $G_{\rho}$ est un espace vectoriel sur lequel on peut définir une norme III || $\left.\right|_{\rho}$ en posant, pour tout $\phi=\sum_{n \geq 0} \phi_{n} \varepsilon^{n} \in G_{\rho}$,

$$
\|\phi\|_{\rho}=\|\phi\|_{\rho}^{\left(R, R^{\prime}\right)}+\left\|\frac{\partial}{\partial y} \phi\right\|_{\rho}^{\left(R, R^{\prime}\right)}+\left\|\frac{\partial^{2}}{\partial y^{2}} \phi\right\|_{\rho}^{\left(R, R^{\prime}\right)} .
$$

De manière analogue à ce qui a été fait pour l'espace $E_{\rho}$, on peut vérifier la propriété de complétude suivante.

Proposition 10. - Muni de la norme $\|\mid\|_{\rho}$, l'espace vectoriel $G_{\rho}$ est un espace de Banach.

On remarque que pour $0<\rho^{\prime}<\rho$, on a une inclusion $G_{\rho} \subset G_{\rho^{\prime}}$ qui est continue, ce qui fait que ces espaces s'organisent en une famille inductive $\left(G_{\rho}\right)_{\rho>0}$ d'espaces de Banach.

Enfin, il faut introduire un dernier type d'espace dont les éléments jouent un rôle analogue à celui des paramètres dans l'approche de la surstabilité par les familles d'équations à $p$ paramètres.

TOME $128-2000-\mathrm{N}^{\circ} 4$ 
DÉFinition 5. - On désigne par $H_{\rho}^{p}$ l'espace vectoriel complexe des séries formelles

$$
f=\sum_{n \geq 1} f_{n} \varepsilon^{n} \in \varepsilon \mathbb{C}[x]_{p}[[\varepsilon]]
$$

dont chaque coefficient $f_{n}$ est un polynôme de degré $<p$ noté

$$
f_{n}=\sum_{j=0}^{p-1} a_{j n} x^{j}
$$

tel que, pour chaque $j=0, \ldots, p-1$, on ait

$$
\sum_{n=1}^{+\infty}\left|a_{j n}\right| \frac{\rho^{n}}{n !}<+\infty
$$

Sur $H_{\rho}^{p}$, on définit une norme ||$_{\rho}$ en posant, pour tout $f=\sum_{n \geq 1}\left(\sum_{j=0}^{p-1} a_{j n} x^{j}\right) \varepsilon^{n}$

$$
|f|_{\rho}=\sum_{j=0}^{p-1}\left(\sum_{n=1}^{+\infty}\left|a_{j n}\right| \frac{\rho^{n}}{n !}\right) .
$$

On peut aussi présenter $H_{\rho}^{p}$ sous la forme de l'ensemble $\mathbb{C}[[\varepsilon]]_{(\rho)}[x]_{p}$ des polynômes de degré strictement inférieur à $p$ à coefficients dans l'ensemble $\mathbb{C}[[\varepsilon]]_{(\rho)}$ des séries formelles $\sum_{n \geq 1} a_{n} \varepsilon^{n} \in \mathbb{C}[[\varepsilon]]$ telles que

$$
\sum_{n=1}^{+\infty}\left|a_{n}\right| \frac{\rho^{n}}{n !}<+\infty
$$

Il est techniquement utile pour la suite que ce nouvel espace $H_{\rho}^{p}$ se réalise comme sous-espace des précédents.

Proposition 11. - Pour chaque $\rho>0$, on a

$$
H_{\rho}^{p}=F_{\rho} \cap \mathbb{C}[x]_{p}[[\varepsilon]]=G_{\rho} \cap\left(\varepsilon \mathbb{C}[x]_{p}[[\varepsilon]]\right)
$$

De plus, sur l'espace $H_{\rho}^{p}$ les normes ||$_{\rho},\|\|_{\rho}$ et $\|\mid\|_{\rho}$ sont équivalentes.

C'est en partie pour obtenir un énoncé aussi simple que la définition initiale de $d(x)$ proposée dans [6] a été modifiée. 
Preuve de la proposition. - Elle résulte des quatres points suivants.

1) Si $f=\sum_{n \geq 1}\left(\sum_{j=0}^{p-1} a_{j n} x^{j}\right) \varepsilon^{n} \in H_{\rho}^{p}$, alors $f \in F_{\rho} \cap \mathbb{C}[x]_{p}[[\varepsilon]]$ et

$$
\|f\|_{\rho} \leq \max \left(1, R^{p-1}\right)|f|_{\rho}
$$

ce qui découle directement de la majoration

$$
\sup _{|x|<R}\left(\left|\sum_{j=0}^{p-1} a_{j n} x^{j}\right|(d(x))^{n}\right) \leq \sum_{j=0}^{p-1} R^{j}\left|a_{j n}\right| .
$$

2) Si $f=\sum_{n \geq 1}\left(\sum_{j=0}^{p-1} a_{j n} x^{j}\right) \varepsilon^{n} \in F_{\rho} \cap \mathbb{C}[x]_{p}[[\varepsilon]]$, alors $f \in H_{\rho}^{p}$ et

$$
|f|_{\rho} \leq\left(\sum_{j=0}^{p-1} r^{-j}\right)\|f\|_{\rho}
$$

En effet, la formule de Cauchy donne $\left|a_{j n}\right| \leq 1 / r^{j} \sup _{|x|=r}\left|f_{n}(x)\right|$. Puisque pour $|x|=r$ on a $\left|f_{n}(x)\right| \leq N_{n}\left(f_{n}\right)$, il vient $\left|a_{j n}\right| \leq 1 / r^{j} N_{n}\left(f_{n}\right)$.

3) Si $f=\sum_{n \geq 1}\left(\sum_{j=0}^{p-1} a_{j n} x^{j}\right) \varepsilon^{n} \in H_{\rho}^{p}$, alors $f \in G \rho \cap\left(\varepsilon \mathbb{C}[x]_{p}[[\varepsilon]]\right)$ et de plus,

$$
\left\|\left.f\left|\|_{\rho} \leq \max \left(1, R^{p-1}\right)\right| f\right|_{\rho}\right.
$$

Cela découle immédiatement de la majoration

$$
\sup _{|x|<R}\left(\left|\sum_{j=0}^{p-1} a_{j n} x^{j}\right|\right) \leq \sum_{j=0}^{p-1} R^{j}\left|a_{j n}\right| .
$$

4) Si $f=\sum_{n \geq 1}\left(\sum_{j=0}^{p-1} a_{j n} x^{j}\right) \varepsilon^{n} \in G_{\rho} \cap\left(\varepsilon \mathbb{C}[x]_{p}[[\varepsilon]]\right)$, alors $f \in H_{\rho}^{p}$ et

$$
|f|_{\rho} \leq\left(\sum_{j=0}^{p-1} R^{-j}\right)\|f\|_{\rho}
$$

En effet, la formule de Cauchy donne $\left|a_{j n}\right| \leq 1 / R^{j}\left\|f_{n}\right\|^{(R)}$. On en déduit

$$
\sum_{n=1}^{+\infty}\left|a_{j n}\right| \frac{\rho^{n}}{n !} \leq \frac{1}{R^{j}} \mid\|f\|_{\rho} .
$$

De cette proposition, il découle que $H_{\rho}^{p}$ est un sous-espace fermé de $F_{\rho}$ et $\operatorname{de} G_{\rho}$.

Corollaire 2. - Muni de la norme ||$_{\rho}$, l'espace vectoriel $H_{\rho}^{p}$ est un espace de Banach. 


\section{Géométrie de l'ensemble des équations surstables}

\subsection{L'ensemble des équations formelles surstables.}

Les espaces considérés jusqu'ici dépendaient tous d'un paramètre $\rho$ réel strictement positif correspondant en gros au type des séries Gevrey qui y interviennent (plus exactement à l'inverse du type). Il faut maintenant s'affranchir de cette contrainte en prenant des limites inductives de ces familles d'espaces lorsque $\rho \rightarrow 0_{+}$. Puisque l'on travaille avec des familles d'espaces $\left(X_{\rho}\right)_{\rho>0}$ qui sont décroissantes pour l'inclusion

$$
\rho<\rho^{\prime} \Longrightarrow X_{\rho^{\prime}} \subset X_{\rho}
$$

on a simplement, au moins au niveau de la structure des ensembles,

$$
\underset{\rho>0}{\lim _{\rho}} X_{\rho}=\bigcup_{\rho>0} X_{\rho}
$$

Cependant, les résultats que nous serons amenés à énoncer prennent tout leur sens dans le cadre des limites inductives.

DÉfinition 6. - On introduit les limites inductives suivantes d'espaces de Banach :

$$
\mathbb{G}=\lim _{\rho>0} G_{\rho}, \quad \mathbb{F}=\underset{\rho>0}{\lim _{\rho}} F_{\rho}, \quad \mathbb{H}^{p}=\underset{\rho>0}{\lim _{\rho}} H_{\rho}^{p}
$$

$\grave{A}$ tout élément $\phi$ de l'espace $\mathbb{G}$ on associe l'équation différentielle $\left(\mathcal{E}_{\phi}^{\prime}\right)$

$$
\varepsilon \frac{d y}{d x}=x^{p} \chi(x) y+\phi(x, y)
$$

Un élément $\phi$ de l'espace $\mathbb{G}$ est une équation formelle surstable si l'équation différentielle $\left(\mathcal{E}_{\phi}^{\prime}\right)$ admet une solution dans $\mathbb{F}$.

On note $\mathbb{S}$ l'ensemble des équations formelles surstables.

On obtient ainsi des espaces de séries formelles Gevrey d'ordre 1 sans contrainte sur le type. En particulier, il est éclairant de préciser la nature de $\mathbb{H}^{p}$. Pour cela, on introduit l'espace $\mathbb{C}[[\varepsilon]]_{1}$ des séries formelles Gevrey d'ordre 1 à coefficients complexes comme étant la limite inductive lorsque $A \rightarrow \infty$ dans $\mathbb{R}_{+}^{*}$

$$
\mathbb{C}[[\varepsilon]]_{1}=\underset{0<A}{\lim _{0<A}} \mathbb{C}[[\varepsilon]]_{(1, A)}
$$

des espaces $\mathbb{C}[[\varepsilon]]_{(1, A)}$ de séries formelles Gevrey d'ordre 1 et de type $A>0$ (cf. [9], [12])

$$
\mathbb{C}[[\varepsilon]]_{(1, A)}=\left\{\sum_{n \geq 0} a_{n} \varepsilon^{n} \in \mathbb{C}[[\varepsilon]] ; \exists C>0, \forall n \in \mathbb{N}\left|a_{n}\right| \leq C, A^{n} n !\right\},
$$


ce dernier étant muni de la norme \|\|$_{(1, A)}$ définie par

$$
\|a\|_{(1, A)}=\sup _{n \geq 0} \frac{\left|a_{n}\right|}{A^{n} n !} \quad \text { pour } \quad a=\sum_{n \geq 0} a_{n} \varepsilon^{n} .
$$

Pour tout $\rho>0$, on a une injection continue

$$
H_{\rho}^{p} \subset \varepsilon \mathbb{C}[[\varepsilon]]_{\left(1, \rho^{-1}\right)}[x]_{p}
$$

de même, pour tout $A>0$ et tout $\rho<1 / A$ on a une injection continue

$$
\varepsilon \mathbb{C}[[\varepsilon]]_{(1, A)}[x]_{p} \subset H_{\rho}^{p}
$$

On en déduit que l'espace $\mathbb{H}^{p}$ est égal à $\varepsilon \mathbb{C}[[\varepsilon]]_{1}[x]_{p}$, lui-même isomorphe à $\left(\varepsilon \mathbb{C}[[\varepsilon]]_{1}\right)^{p}$.

On peut maintenant donner une nouvelle interprétation du théorème de point fixe précédent (théorème 1); étant donné $\phi \in \mathbb{G}$, il existe un unique $h \in \mathbb{F}$ tel que

$$
h=\varepsilon \frac{\partial}{\partial x} \mathcal{R}_{p} h-\widetilde{\phi}\left(\mathcal{R}_{p} h\right)
$$

Or tout $h \in \mathbb{F}$ se décompose de manière unique sous la forme $h=x^{p} \chi(x) f+g$ avec $f \in \mathbb{F}$ et $g \in \mathbb{H}^{p}$ (où $f=\mathcal{R}_{p} h$ et $g=\mathcal{T}_{p} h$ ). On en déduit l'existence et l'unicité de $f \in \mathbb{F}$ et $g \in \mathbb{H}^{p}$ tels que

$$
\varepsilon \frac{\partial}{\partial x} f=x^{p} \chi(x) f+\widetilde{\phi}(f)+g .
$$

Introduisant $\psi=\phi+g$ qui est un élément de $\mathbb{G}$, on voit que cette dernière équation s'identifie à $\left(\mathcal{E}_{\psi}^{\prime}\right)$ (confer l'équation (8))

$$
\varepsilon \frac{d y}{d x}=x^{p} \chi(x) y+\psi(x, y)
$$

d'où le résultat suivant.

ThÉORÈme 2. - Pour tout $\phi \in \mathbb{G}$, il existe une unique équation formelle surstable $\psi$ appartenant au sous-espace affine $\phi+\mathbb{H}^{p}$ de $\mathbb{G}$. De plus, l'équation différentielle $\mathcal{E}_{\psi}^{\prime}$ associée à $\psi$ admet une unique solution dans $\mathbb{F}$.

Autrement dit, l'ensemble $\mathbb{S}$ des équations surstables formelles rencontre en un unique point tout sous-espace affine de direction $\mathbb{H}^{p}$ de l'espace $\mathbb{G}$. On peut s'interroger maintenant sur la géométrie locale de $\mathbb{S}$. Pour cela, on va caractériser $\mathbb{S}$ comme le lieu des zéros d'une application et étudier la régularité de cette dernière.

TOME $128-2000-\mathrm{N}^{\circ} 4$ 
On introduit l'application $\mathcal{K}: \mathbb{G} \rightarrow \mathbb{H}^{p}$ qui, à $\phi \in \mathbb{G}$, fait correspondre l'unique $g \in \mathbb{H}^{p}$ intervenant dans l'équation (10). D'après ce qui précède, l'ensemble $\mathbb{S}$ des équations surstables formelles est égale à $\mathcal{K}^{-1}(0)$. Puis on considère l'application $\mathcal{L}: \mathbb{G} \rightarrow \mathbb{F}$ qui à $\phi \in \mathbb{G}$ fait correspondre $h \in \mathbb{F}$ tel que l'équation (9) soit satisfaite. On remarque que $\mathcal{K}=\mathcal{T}_{p} \circ \mathcal{L}$, ce qui fait que l'étude de la régularité de $\mathcal{K}$ est ramenée à celle de $\mathcal{L}$.

On remarque que, pour $\phi \in \mathbb{G}$, l'élément $\mathcal{L}(\phi)$ de $\mathbb{F}$ est la solution de l'équation (9) interprétée comme une équation en $h$. En vue d'appliquer le théorème des fonctions implicites, on est amené à étudier, pour $\rho>0$ fixé, l'application

$$
\begin{aligned}
\mathcal{G}: G_{\rho} \times B_{F_{\rho}}\left(0, K_{p}^{-1} R^{\prime}\right) & \longrightarrow F_{\rho}, \\
(\phi, h) & \longmapsto h-\varepsilon \frac{\partial}{\partial x} \mathcal{R}_{p} h+\widetilde{\phi}\left(\mathcal{R}_{p} h\right),
\end{aligned}
$$

où $B_{F_{\rho}}\left(0, K_{p}^{-1} R^{\prime}\right)$ désigne la boule ouverte de centre 0 et de rayon $K_{p}^{-1} R^{\prime}$ dans l'espace $F_{\rho}$ avec $K_{p}=(2 / \rho)^{p}\|1 / \chi\|^{(R)}$ (confer $\left.\S 5\right)$.

LeMme 5. - L'application $\mathcal{G}$ est de classe $C^{1}$ sur $G_{\rho} \times B_{F_{\rho}}\left(0, K_{p}^{-1} R^{\prime}\right)$ et ses différentielles partielles en tout point $\left(\phi^{0}, h^{0}\right) \in G_{\rho} \times B_{F_{\rho}}\left(0, K_{p}^{-1} R^{\prime}\right)$ sont

$$
\begin{array}{ll}
D_{1}(\mathcal{G})\left(\phi^{0}, h^{0}\right): G_{\rho} \longrightarrow F_{\rho}, \quad & \phi \longmapsto \widetilde{\phi}\left(\mathcal{R}_{p} h^{0}\right) ; \\
D_{2}(\mathcal{G})\left(\phi^{0}, h^{0}\right): F_{\rho} \longrightarrow F_{\rho}, & h \longmapsto h-\varepsilon \frac{\partial}{\partial x} \mathcal{R}_{p} h+\frac{\partial}{\partial y} \phi^{0}\left(\mathcal{R}_{p} h^{0}\right) \mathcal{R}_{p} h .
\end{array}
$$

Preuve du lemme 5. - L'application partielle

$$
\phi \mapsto \mathcal{G}\left(\phi, h^{0}\right)=h^{0}-\varepsilon \frac{\partial}{\partial x} \mathcal{R}_{p} h^{0}+\widetilde{\phi}\left(\mathcal{R}_{p} h^{0}\right)
$$

est affine de partie linéaire l'application

$$
G_{\rho} \longrightarrow F_{\rho}, \quad \phi \longmapsto \widetilde{\phi}\left(\mathcal{R}_{p} h^{0}\right) .
$$

Puisque, d'après la proposition $\left.8, \| \widetilde{\phi}\left(\mathcal{R}_{p} h^{0}\right)\right)\left\|_{\rho} \leq R^{\prime} /\left(R^{\prime}-\left\|\mathcal{R}_{p} h^{0}\right\|_{\rho}\right)\right\| \phi \|_{\rho}$, cette application linéaire est bien continue de norme inférieur ou égale à $R^{\prime} /\left(R^{\prime}-K_{p}\left\|h^{0}\right\|_{\rho}\right)$, d'où le résultat annoncé sur la différentielle $D_{1}(\mathcal{G})\left(\phi^{0}, h^{0}\right)$.

La différence $D_{1}(\mathcal{G})\left(\phi^{1}, h^{1}\right)-D_{1}(\mathcal{G})\left(\phi^{0}, h^{0}\right)$ étant l'application linéaire

$$
\phi \mapsto \widetilde{\phi}\left(\mathcal{R}_{p} h^{1}\right)-\widetilde{\phi}\left(\mathcal{R}_{p} h^{0}\right),
$$

la continuité de $D_{1}(\mathcal{G})$ découle des inégalités

$$
\begin{gathered}
\left\|\widetilde{\phi}\left(\mathcal{R}_{p} h^{1}\right)-\widetilde{\phi}\left(\mathcal{R}_{p} h^{0}\right)\right\|_{\rho} \leq \sup _{h \in\left[h^{0}, h^{1}\right]}\left\|D \widetilde{\phi}\left(\mathcal{R}_{p} h\right)\right\|_{\rho} \cdot\left\|\mathcal{R}_{p} h^{1}-\mathcal{R}_{p} h^{0}\right\|_{\rho}, \\
\left\|D \widetilde{\phi}\left(\mathcal{R}_{p} h\right)\right\|_{\rho} \leq\left\|\frac{\partial}{\partial y} \phi\left(\mathcal{R}_{p} h\right)\right\|_{\rho} \leq \frac{R^{\prime}}{R^{\prime}-K_{p}\|h\|_{\rho}}\|\phi\|_{\rho}
\end{gathered}
$$

qui proviennent des propositions 8 et 9 . 
D'après la même proposition 9, l'application partielle

$$
h \longmapsto \mathcal{G}\left(\phi^{0}, h\right)=h-\varepsilon \frac{\partial}{\partial x} \mathcal{R}_{p} h+\widetilde{\phi^{0}}\left(\mathcal{R}_{p} h\right)
$$

est différentiable en $h^{0}$ de différentielle l'application $D_{2}(\mathcal{G})\left(\phi^{0}, h^{0}\right)$ donnée dans l'énoncé. De plus

$$
\left\|D_{2}(\mathcal{G})\left(\phi^{1}, h^{1}\right)-D_{2}(\mathcal{G})\left(\phi^{0}, h^{0}\right)\right\|_{\rho} \leq K_{p}\left\|\widetilde{\frac{\partial}{\partial y}} \phi^{1}\left(\mathcal{R}_{p} h^{1}\right)-\widetilde{\frac{\partial}{\partial y}} \phi^{0}\left(\mathcal{R}_{p} h^{0}\right)\right\|_{\rho}
$$

avec $K_{p}=(2 / r)^{p}\|1 / \xi\|^{(R)}$ un majorant de $\left\|\mathcal{R}_{p}\right\|_{\rho}$. Puisque

$$
\begin{aligned}
& \left\|\widetilde{\frac{\partial}{\partial y} \phi^{1}}\left(\mathcal{R}_{p} h^{1}\right)-\widetilde{\frac{\partial}{\partial y}} \phi^{0}\left(\mathcal{R}_{p} h^{0}\right)\right\|_{\rho} \\
& \leq\left\|\widetilde{\frac{\partial}{\partial y} \phi^{1}}\left(\mathcal{R}_{p} h^{1}\right)-\widetilde{\frac{\partial}{\partial y}} \phi^{1}\left(\mathcal{R}_{p} h^{0}\right)\right\|_{\rho}+\left\|\widetilde{\frac{\partial}{\partial y} \phi^{1}}\left(\mathcal{R}_{p} h^{0}\right)-\widetilde{\frac{\partial}{\partial y}} \phi^{0}\left(\mathcal{R}_{p} h^{0}\right)\right\|_{\rho}
\end{aligned}
$$

et en utilisant à nouveau les prop. 8 et 9 , on voit que $D_{2}(\mathcal{G})$ est continue.

LEMmE 6. - Soient $\left.\rho_{0}>0, \alpha \in\right] 0,1\left[\right.$ et $\left(\phi^{0}, h^{0}\right) \in G_{\rho_{0}} \times F_{\rho_{0}}$ tels que $\mathcal{G}\left(\phi^{0}, h^{0}\right)=0$. Alors, il existe $\left.\left.\rho_{1} \in\right] 0, \rho_{0}\right]$ tel que

$$
\left\|D_{2}(\mathcal{G})\left(\phi_{,}^{0}, h^{0}\right)-\mathrm{id}_{F_{\rho}}\right\|_{\rho}<\alpha
$$

pour tout $\left.\rho \in] 0, \rho_{1}\right]$. En particulier, $D_{2}(\mathcal{G})\left(\phi^{0}, h^{0}\right)$ est un automorphisme de l'espace de Banach $F_{\rho}$ pour tout $\left.\left.\rho \in\right] 0, \rho_{1}\right]$.

Preuve du lemme 6. - Pour tout $\left.\rho \in] 0, \rho_{0}\right]$, l'élément $h^{0}$ est un point fixe dans $F_{\rho}$, de l'opérateur $\mathcal{F}^{0}: h \mapsto \varepsilon \partial / \partial x \mathcal{R}_{p} h-\widetilde{\phi^{0}}\left(\mathcal{R}_{p} h\right)$ et, compte tenu du lemme 5

$$
D_{2}(\mathcal{G})\left(\phi^{0}, h^{0}\right)-\operatorname{id}_{F_{\rho}}=-D\left(\mathcal{F}^{0}\right)\left(h^{0}\right) .
$$

Le résultat découle donc du lemme 4 et du théorème 1 de point fixe (ainsi que de sa démonstration).

On en sait maintenant assez sur l'application $\mathcal{G}$ pour décrire la géométrie de l'ensemble $\mathbb{S}$ des équations formelles surstables.

THÉORÈme 3. - Pour toute équation formelle surstable $\phi^{0}$, il existe $\rho_{1} \in \mathbb{R}_{+}^{*}$ tel que $\phi^{0} \in G_{\rho_{1}}$ et que, pour tout $\left.\left.\rho \in\right] 0, \rho_{1}\right]$, l'ensemble $\mathbb{S} \cap G_{\rho}$ des équations formelles surstables appartenant à $G_{\rho}$ soit, localement au voisinage de $\phi^{0}$ dans $G_{\rho}$, une sous-variété différentiable de classe $C^{1}$ de l'espace de Banach $G_{\rho}$ transversale dans ce dernier au sous-espace affine passant par $\phi^{0}$ et de direction $H_{\rho}^{p}$.

On peut convenir de condenser l'information contenue dans cet énoncé de la manière suivante.

TOME $128-2000-\mathrm{N}^{\circ} 4$ 
L'ensemble $\mathbb{S}$ des équations formelles surstables est inductivement une sousvariété différentiable de classe $C^{1}$ de l'espace $\mathbb{G}=\lim _{\rho>0} G_{\rho}$, partout transversale à la direction du sous-espace $\mathbb{H}^{p}=\lim _{\rho>0} H_{\rho}^{p}$.

Preuve du théorème 3. - Soit $\phi^{0} \in \mathbb{G}$ une équation surstable formelle. Il existe donc $f^{0} \in \mathbb{F}$ qui est solution de l'équation différentielle $\left(\mathcal{E}_{\phi^{0}}^{\prime}\right)$. Cela revient à dire que $h^{0}=x^{p} \chi f^{0}$ satisfait l'équation (9), c'est-à-dire vérifie $\mathcal{G}\left(\phi^{0}, h^{0}\right)=0$. D'après les lemmes 5 et 6 , on peut appliquer le théorème des fonctions implicites à l'équation $\mathcal{G}(\phi, h)=0$ au voisinage de $\left(\phi^{0}, h^{0}\right)$ dans $F_{\rho}$ pour $\rho$ assez petit. On en déduit qu'il existe $\rho^{\prime}>0$ tel que, $\left(\phi^{0}, f^{0}\right) \in G_{\rho^{\prime}} \times F_{\rho^{\prime}}$ et que, pour tout $\left.\left.\rho \in\right] 0, \rho^{\prime}\right]$, l'application $\mathcal{K}=\mathcal{T}_{p} \circ \mathcal{L}$ soit, au voisinage de $\phi^{0}$ dans $G_{\rho}$, une fonction à valeurs dans $H_{\rho}^{p}$ de classe $C^{1}$ avec une différentielle donnée par

$$
D(\mathcal{K})(\phi)=-\mathcal{T}_{p} \circ D_{2}(\mathcal{G})(\phi, h)^{-1} \circ D_{1}(\mathcal{G})(\phi, h)
$$

Pour tout $g \in H_{\rho}^{p}$, on a $\widetilde{g}\left(\mathcal{R}_{p} h^{0}\right)=g$ du fait que $g$ ne dépend pas de la variable $y$, d'où

$$
D(\mathcal{K})\left(\phi^{0}\right) \cdot g=-\mathcal{T}_{p}\left[\left(D_{2}(\mathcal{G})\left(\phi^{0}, h^{0}\right)\right)^{-1} \cdot g\right]
$$

D'après le lemme 6 , on peut supposer que $\left\|D_{2}(\mathcal{G})\left(\phi^{0}, h^{0}\right)-\operatorname{id}_{F_{\rho}}\right\|_{\rho}<\alpha$ avec $\alpha$ arbitrairement petit. De plus, on peut exprimer l'inverse de $D_{2}(\mathcal{G})\left(\phi^{0}, h^{0}\right)$ de la manière suivante

$$
\left(D_{2}(\mathcal{G})\left(\phi^{0}, h^{0}\right)\right)^{-1}=\operatorname{id}_{F_{\rho}}+\sum_{n=1}^{+\infty}\left(\operatorname{id}_{F_{\rho}}-D_{2}(\mathcal{G})\left(\phi^{0}, h^{0}\right)\right)^{n} .
$$

Comme $g \in H_{\rho}^{p}$, on a $\mathcal{T}_{p}(g)=g$, d'où

$$
D(\mathcal{K})\left(\phi^{0}\right) \cdot g=-\left(\operatorname{id}_{H_{\rho}^{p}}-\mathcal{T}_{p} \sum_{n=1}^{+\infty}\left(\operatorname{id}_{F_{\rho}}-D_{2}(\mathcal{G})\left(\phi^{0}, h^{0}\right)\right)^{n}\right) \cdot g
$$

et on peut choisir $\alpha$ assez petit de sorte que

$$
\left\|\mathcal{T}_{p} \sum_{n=1}^{+\infty}\left(\operatorname{id}_{F_{\rho}}-D_{2}(\mathcal{G})\left(\phi^{0}, h^{0}\right)\right)^{n}\right\|_{\rho}<1
$$

ce qui fait que, en restriction à l'espace $H_{\rho}^{p}$, l'application $D(\mathcal{K})\left(\phi^{0}\right)$ est un automorphisme de $H_{\rho}^{p}$.

Il en découle que l'application $\mathcal{K}$ est une submersion au voisinage de $\phi^{0}$ dans $G_{\rho}$ et que $\operatorname{ker}\left(D(\mathcal{K})\left(\phi^{0}\right)\right)$ est transverse à $H_{\rho}^{p}$. 


\subsection{L'ensemble des équations $\mathcal{C}$-surstables.}

Les résultats du sous-paragraphe précédent constituent la partie principale de cette étude. On a vu qu'ils concernent le problème de la surstabilité dans des espaces de séries formelles à coefficients holomorphes. On peut maintenant en déduire quelques conséquences pour la surstabilité dans des espaces de fonctions analytiques. Cela peut se faire en s'appuyant sur le procédé de sommation de Borel-Laplace tronquée et sur la notion de quasi-solution développés en particulier dans [6].

Avec les notations du paragraphe 2 , on fixe maintenant $\left(r, R, R^{\prime}\right) \in\left(\mathbb{R}_{+}^{*}\right)^{3}$ de sorte que $r<R$ et que l'adhérence dans $\mathbb{C}^{2}$ de $D(0, R) \times D\left(0, R^{\prime}\right)$ soit incluse dans le voisinage ouvert $U_{1}$ de 0 dans $\mathbb{C}^{2}$ qui est l'image de $U_{0}$ par l'isomorphisme analytique $(\xi, u) \mapsto\left(\xi-\xi_{0}, u-\varphi_{0}(\xi)\right)$. Pour ce choix, on considère les espaces $\mathbb{F}$, $\mathbb{G}$ et $\mathbb{H}$ définis dans les paragraphes précédent et l'ensemble $\mathbb{S} \subset \mathbb{G}$ des solutions surstables formelles.

Étant donné $F \in \mathcal{A}\left(W_{0}, \varphi_{0}, \chi, p\right)$ de développement asymptotique $\sum_{n \geq 0} F_{n} \varepsilon^{n}$, on note $\pi(F)$ la série formelle $\sum_{n \geq 0} \phi_{n} \varepsilon^{n}$ ainsi définie :

- $\phi_{0}$ est la restriction à $D(0, R) \times D\left(0, R^{\prime}\right)$ de la fonction

$$
(x, y) \longmapsto F_{0}\left(\xi_{0}+x, \varphi_{0}\left(\xi_{0}+x\right)+y\right)-x^{p} \chi(x) y ;
$$

- pour chaque $n>0, \phi_{n}$ est la restriction à $D(0, R) \times D\left(0, R^{\prime}\right)$ de la fonction

$$
(x, y) \longmapsto F_{n}\left(\xi_{0}+x, \varphi_{0}\left(\xi_{0}+x\right)+y\right) .
$$

ThÉORÈme 4. - Soit $F \in \mathcal{A}\left(W_{0}, \varphi_{0}, \chi, p\right)$. Alors $\pi(F)$ appartient à $\mathbb{G}$. De plus, $F$ est une équation $\mathcal{C}$-surstable si et seulement si $\pi(F)$ est une équation formelle surstable.

Preuve du théorème 4. - La démonstration de la première affirmation est une généralisation au cas à paramètre d'une propriété basique en asymptotique exacte [10], [13].

Étant donné un secteur ouvert $S$ de sommet 0 dans $\mathbb{C}$ tel que $S \Subset S_{0}$, il existe des constantes réelles positives $A$ et $B$ de sorte que, pour tout $N \in \mathbb{N}^{*}$ et pour tout $(\xi, u, \varepsilon) \in U_{0} \times S$

$$
\left|F(\xi, u, \varepsilon)-\sum_{n=0}^{N-1} F_{n}(\xi, u) \varepsilon^{n}\right| \leq A B^{N} N !|\varepsilon|^{N} .
$$

On en déduit que $F_{n}$ est uniformément majoré par $A B^{N} N$ ! sur $U_{0}$ et il en est de même pour $\phi_{n}$ sur $U_{1}$. On choisit alors $\rho \in \mathbb{R}_{+}^{*}$ tel que $\rho B<1$. Alors, avec les notations de la définition 4 ,

$$
\begin{aligned}
& \|\pi(F)\|_{\rho}^{\left(R, R^{\prime}\right)}<+\infty, \quad\left\|\frac{\partial}{\partial y} \pi(F)\right\|_{\rho}^{\left(R, R^{\prime}\right)}<+\infty, \quad\left\|\frac{\partial^{2}}{\partial y^{2}} \pi(F)\right\|_{\rho}^{\left(R, R^{\prime}\right)}<+\infty \\
& \text { томе } 128-2000-\mathrm{N}^{\circ} 4
\end{aligned}
$$


les deux dernières relations nécessitant l'utilisation des inégalités de Cauchy. Enfin, par construction, les fonctions $x \mapsto \phi_{0}(x, 0)$ et $x \mapsto \frac{\partial}{\partial y} \phi_{0}(x, 0)$ sont nulles, ce qui fait que $\pi(F) \in \mathbb{G}$.

Si $F$ est une équation $\mathcal{C}$-surstable, alors $F$ admet une solution $\mathcal{C}$-formelle constituée par le développement asymptotique d'une solution $\mathcal{C}$-surstable. On en déduit que $\mathcal{K}(\pi(F))=0$ et donc que $\pi(F)$ est une équation surstable formelle.

La réciproque de cette dernière propriété repose sur la sommation de BorelLaplace tronquée ainsi que sur la notion de quasi-solution [6], [12]. Si $\pi(F)$ est une équation surstable formelle, l'équation $\left(\mathcal{E}_{\pi(F)}^{\prime}\right)$ admet une solution $f \in \mathbb{F}$. En appliquant à $f$ une sommation de Borel-Laplace tronquée, on obtient une quasi-solution de $\left(\mathcal{E}_{F}\right)$ d'où l'on déduit une solution $\mathcal{C}$-surstable.

En conséquence, $\pi$ est une application de $\mathcal{A}\left(W_{0}, \varphi_{0}, \chi, p\right)$ dans $\mathbb{G}$ et l'ensemble $\mathbb{S}_{\mathcal{C}}$ des équations $\mathcal{C}$-surstables est égal à $\pi^{-1}(\mathbb{S})$, c'est-à-dire à l'image réciproque par $\pi$ d'un ensemble qui est inductivement une sous-variété de classe $C^{1}$ dans $\mathbb{G}$ et transverse à $\mathbb{H}^{p}$. Pour compléter le sens de cette description, il est bon de noter que $\pi$ est une application linéaire et que, d'après une propriété classique en asymptotique Gevrey [8], [9], [11], [14], son noyau est l'ensemble des éléments de $\mathcal{A}\left(W_{0}, \varphi_{0}, \chi, p\right)$ qui sont exponentiellement décroissants d'ordre 1 lorsque $\varepsilon$ tend vers 0 . Ainsi, l'ensemble $\mathbb{S}_{\mathcal{C}}$ est une sorte d'épaississement exponentiellement petit d'une variété; par analogie avec la notion de quasi-solution, on pourrait dire que $\mathbb{S}_{\mathcal{C}}$ est une quasi-variété. On trouve dans [6] un résultat de même type dans l'espace de paramètres pour une famille d'équations à nombre fini de paramètres complexes.

\section{BIBLIOGRAPHIE}

[1] Balser (W.). - From Divergent Power Series to Analytic Function, Lecture Notes in Mathematics 1582. - Springer-Verlag, Berlin Heidelberg, 1994.

[2] Benoît (E.), Callot (J.-L.), Diener (F.), Diener (M.). - Chasse aux canards, Collect. Math., t. 31, 3, 1981, p. 37-119.

[3] Benoît (E.), Fruchard (A.), Schäfke (R.), Wallet (G.). - Overstability : toward a global study, C. R. Acad. Sci. Paris, t. 326, Série I, 1998, p. $873-878$.

[4] Benoît (E.), Fruchard (A.), Schäfke (R.), Wallet (G.). - Solutions surstables des équations différentielles complexes lentes-rapides à points tournants, Ann. Fac. Sci. Toulouse, t. 7, 4, 1998, p. 627-658. 
[5] Canalis-Durand (M.). - Solution formelle Gevrey d'une équation différentielle singulièrement perturbée, Asymp. Anal., t. 8, 1994, p. 185-216.

[6] Canalis-Durand (M.), Ramis (J.-P.), Schäfke (R.), Sibuya (Y.).- Gevrey solutions of singularly perturbed differential equations, à paraître dans J. reine angew. Math, 1999.

[7] Fruchard (A.), Schäfke (R.). - Surstabilité et résonance, prépublication, 1999 .

[8] Malgrange (B.). - Resommation des séries divergentes, Expo. Math., t. 13, 1995, p. 163-222.

[9] Ramis (J.-P.). - Dévissage Gevrey, Astérisque, Soc. Math. de France, t. 5960, 1978, p. 173-204.

[10] Ramis (J.-P.). - Les derniers travaux de Jean Martinet, Ann. Inst. Fourier, t. 42, 1-2, 1992, p. 15-47.

[11] Ramis (J.-P.). - Séries divergentes et théories asymptotiques, Panoramas et synthèses I, Soc. Math. de France, 1993.

[12] Ramis (J.-P.), Schäfke (R.).- Gevrey Separation of fast and slow variables, Nonlinearity, t. 9, 1996, p. 353-384.

[13] Sснӓғке (R.). - On a theorem of Sibuya, Letter to Sibuya, 1991.

[14] Tougeron (J.-C.). - An introduction to the theory of Gevrey expansions and to the Borel-Laplace transform with some applications, Polycopié d'un cours fait à Rennes et à Toronto, 1989-1990.

[15] Wallet (G.). - Surstabilité pour une équation différentielle analytique en dimension 1, Ann. Inst. Fourier, t. 40, 3, 1990, p. 557-595.

[16] Wallet (G.). - Singularité analytique et perturbation singulière en dimension 2, Bull. Soc. Math. France, t. 122, 1994, p. 185-208.

TOME $128-2000-\mathrm{N}^{\circ} 4$ 